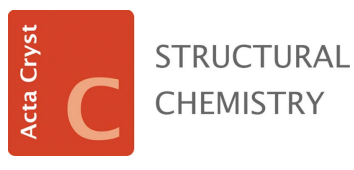

ISSN 2053-2296

Received 27 April 2021

Accepted 16 June 2021

Edited by D. R. Turner, University of Monash, Australia

Keywords: heterocycle; triazole; tautomerism; elusive tautomer; crystal structure; zinc; copper.

CCDC references: 2090148; 2090147

Supporting information: this article has supporting information at journals.iucr.org/C

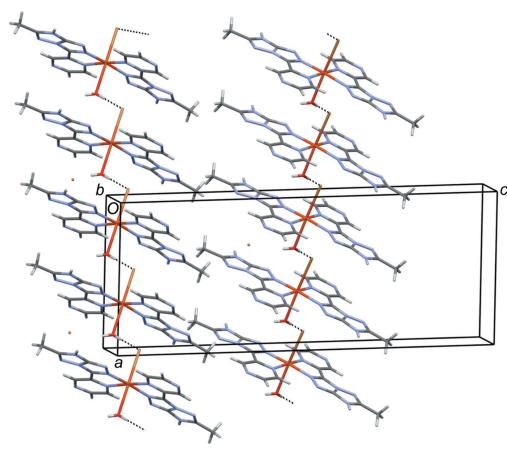

(c) (1) OPEN () ACCESS

\section{Stabilization of an elusive tautomer by metal coordination}

\author{
Emmanuele Parisi and Roberto Centore*
}

Dipartimento di Scienze Chimiche, Università degli Studi di Napoli 'Federico II', Complesso di Monte S. Angelo, Via Cinthia, 80126 Napoli, Italy. *Correspondence e-mail: roberto.centore@unina.it

The solid-state isolation of the different tautomers of a chemical compound can be a challenging problem. In many cases, tautomers with an energy very close to the most stable one cannot be isolated (elusive tautomers). In this article, with reference to the 4-methyl-7-(pyrazin-2-yl)- $2 H$-[1,2,4]triazolo[3,2-c][1,2,4]triazole ligand, for which the elusive $3 H$-tautomer has an energy only $1.4 \mathrm{kcal} \mathrm{mol}^{-1}$ greater than the most stable $2 H$ form, we show that metal complexation is a successful and reliable way for stabilizing the elusive tautomer. We have prepared two complexes of the neutral ligand with $\mathrm{CuBr}_{2}$ and $\mathrm{ZnBr}_{2}$, namely, aquabromidobis[4-methyl-7-(pyrazin-2-yl)-3H-[1,2,4]triazolo[3,2-c][1,2,4]triazole]copper(II) bromide trihydrate, $\left[\mathrm{CuBr}\left(\mathrm{C}_{8} \mathrm{H}_{7} \mathrm{~N}_{7}\right)_{2}\left(\mathrm{H}_{2} \mathrm{O}\right)\right] \mathrm{Br} \cdot 3 \mathrm{H}_{2} \mathrm{O}$, and dibromido[4-methyl-7-(pyrazin-2-yl)-2H-[1,2,4]triazolo[3,2-c][1,2,4]triazole][4-methyl-7(pyrazin-2-yl)-3H-[1,2,4]triazolo[3,2-c][1,2,4]triazole]zinc(II) monohydrate, [Zn$\left.\mathrm{Br}_{2}\left(\mathrm{C}_{8} \mathrm{H}_{7} \mathrm{~N}_{7}\right)_{2}\right] \cdot \mathrm{H}_{2} \mathrm{O}$. The $\mathrm{X}$-ray analysis shows that, in both cases, the elusive $3 \mathrm{H}$-tautomer is present. The results of the crystallographic analysis of the two complexes reflect the different coordination preferences of $\mathrm{Cu}^{\mathrm{II}}$ and $\mathrm{Zn}^{\mathrm{II}}$. The copper(II) complex is homotautomeric as it only contains the elusive $3 \mathrm{H}$ tautomer of the ligand. The complex can be described as octahedral with tetragonal distortion. Two $3 H$-triazolotriazole ligands are bis-chelated in the equatorial plane, while a water molecule and a bromide ion in elongated axial positions complete the coordination environment. The zinc(II) complex, on the other hand, is heterotautomeric and contains two bromide ions and two monodentate ligand molecules, one in the $2 \mathrm{H}$-tautomeric form and the other in the $3 H$-tautomeric form, both coordinated to the metal in tetrahedral geometry. The observation of mixed-tautomer complexes is unprecedented for neutral ligands. The analysis of the X-ray molecular structures of the two complexes allows the deduction of possible rules for a rational design of mixed-tautomer complexes.

\section{Introduction}

Tautomers are structural isomers in ready equilibrium between each other (McNaught \& Wilkinson, 1997). They are intriguing chemical systems that, in a certain sense, can be considered as 'living molecules'. In fact, because of the equilibrium they undergo, the relative amounts of the different forms in solution can be altered by physical or chemical factors (temperature, solvent, $\mathrm{pH}$, metal ions, etc.) through the laws of chemical equilibrium. Tautomers have been central in chemistry since the early work of Berzelius on cyanic and cyanuric acids in 1832, and the discovery of keto-enol tautomerism by Erlenmeyer in 1880 (Taylor et al., 2014). The issue of tautomerism has been fundamental in many turning points of research in chemistry. For instance, it was fundamental in the discovery of the structure of DNA by Watson \& Crick (1953a), which relies on the keto-tautomeric forms of purine and pyrimidine bases, and in their seminal hypothesis (Watson \& Crick, 1953b) that noncanonical tautomeric forms of the 
Table 1

Experimental details.

Experiments were carried out with Mo $K \alpha$ radiation using a Bruker-Nonius KappaCCD diffractometer. Absorption was corrected for by multi-scan methods (SADABS; Bruker, 2001). H atoms were treated by a mixture of independent and constrained refinement.

\begin{tabular}{|c|c|c|}
\hline & 1 & 2 \\
\hline \multicolumn{3}{|l|}{ Crystal data } \\
\hline Chemical formula & {$\left[\mathrm{CuBr}\left(\mathrm{C}_{8} \mathrm{H}_{7} \mathrm{~N}_{7}\right)_{2}\left(\mathrm{H}_{2} \mathrm{O}\right)\right] \mathrm{Br} \cdot 3 \mathrm{H}_{2} \mathrm{O}$} & {$\left[\mathrm{ZnBr}_{2}\left(\mathrm{C}_{8} \mathrm{H}_{7} \mathrm{~N}_{7}\right)_{2}\right] \cdot \mathrm{H}_{2} \mathrm{O}$} \\
\hline$M_{\mathrm{r}}$ & 697.83 & 645.62 \\
\hline Crystal system, space group & Monoclinic, $C c$ & Triclinic, $P \overline{1}$ \\
\hline Temperature (K) & 173 & 293 \\
\hline$a, b, c(\AA)$ & $11.062(4), 8.369$ (3), 27.406 (6) & $8.396(2), 12.305(3), 12.724(3)$ \\
\hline$\alpha, \beta, \gamma\left({ }^{\circ}\right)$ & $90,92.44(3), 90$ & $112.53(2), 107.78(3), 92.16(2)$ \\
\hline$V\left(\AA^{3}\right)$ & $2534.9(14)$ & $1138.1(5)$ \\
\hline$Z$ & 4 & 2 \\
\hline$\mu\left(\mathrm{mm}^{-1}\right)$ & 4.07 & 4.63 \\
\hline Crystal size $(\mathrm{mm})$ & $0.50 \times 0.20 \times 0.20$ & $0.40 \times 0.30 \times 0.20$ \\
\hline \multicolumn{3}{|l|}{ Data collection } \\
\hline$T_{\min }, T_{\max }$ & $0.250,0.478$ & $0.270,0.433$ \\
\hline $\begin{array}{l}\text { No. of measured, independent and observed } \\
\quad[I>2 \sigma(I)] \text { reflections }\end{array}$ & $8682,4870,4563$ & $12306,5090,3636$ \\
\hline$R_{\text {int }}$ & 0.036 & 0.042 \\
\hline$(\sin \theta / \lambda)_{\max }\left(\AA^{-1}\right)$ & 0.651 & 0.649 \\
\hline \multicolumn{3}{|l|}{ Refinement } \\
\hline$R\left[F^{2}>2 \sigma\left(F^{2}\right)\right], w R\left(F^{2}\right), S$ & $0.033,0.081,1.04$ & $0.047,0.136,1.09$ \\
\hline No. of reflections & 4870 & 5090 \\
\hline No. of parameters & 367 & 315 \\
\hline No. of restraints & 13 & 2 \\
\hline$\Delta \rho_{\max }, \Delta \rho_{\min }\left(\mathrm{e} \AA^{-3}\right)$ & $0.60,-0.86$ & $0.70,-0.74$ \\
\hline Absolute structure & Refined as an inversion twin & - \\
\hline Absolute structure parameter & $0.502(12)$ & - \\
\hline
\end{tabular}

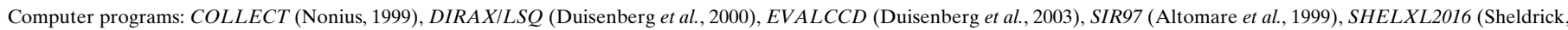
2015), ORTEP-3 for Windows (Farrugia, 2012), Mercury (Macrae et al., 2020) and WinGX (Farrugia, 2012).

bases could be involved in mutagenesis (Goodman, 1995; Wang et al., 2011). Now the relevance of tautomers is increasingly recognized in many fields of applied chemistry, including drug design (Martin, 2009) and materials chemistry (Bussetti et al., 2014; Horiuchi et al., 2017). In the realm of coordination chemistry, tautomerism can also be a relevant issue, because some classes of ligands show tautomerism. As an example, keto-enol tautomerism is shown in aminonaphthol derivatives (Deneva et al., 2019), in dihydroxyquinolines (Todorov et al., 2012) and in aroylhydrazine ligands (Borbone et al., 2004), while thione-thiol tautomerism is present in dithiocarbazate ligands (Takjoo \& Centore, 2013). In most of these cases, the common feature is that only one tautomeric form acts as the ligand and is found in the complexes.<smiles>[R]C1=Nn2cc([R])nc2N([2H])N1C</smiles>

$2 \mathrm{H}$<smiles>[R]c1nn2c([R])nnc2[nH]1</smiles>

$3 H$

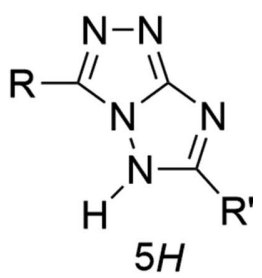

$5 H$
Figure 1

Relevant chemical diagrams for the $[1,2,4]$ triazolo[3,2-c] [1,2,4]triazole system: the three tautomers of the neutral form (the adopted atom numbering of the heterobicycle is indicated only for the $2 \mathrm{H}$-tautomer).
In the realm of fused-ring heteroaromatic systems that we have studied over the years (Centore et al., 1996, 1999; Ambrosanio et al., 1999), we have found in [1,2,4] triazolo[3,2-c]$[1,2,4]$ triazole a heterocyclic system with a rich tautomeric behaviour (Centore et al., 2013, 2015, 2017; Fusco et al., 2018).

We have found that the relative energy of the three tautomers of the neutral molecule (Fig. 1) can be significantly modulated by acting on the electronic character of the substituents and on the polarity of the solvent. In all the cases investigated, the energy trend of the tautomers is $E(2 H)<$ $E(3 H)<<E(5 H)$; in particular, while the predicted energy of the $5 H$-tautomer is always prohibitive $\left(+10.8 \mathrm{kcal} \mathrm{mol}^{-1}\right.$ with<smiles>Cc1nc2[nH]nc(-c3cnccn3)n2n1</smiles>

(a)

$$
\begin{aligned}
& \mathrm{Cu}(\mathrm{TT} 9)_{2} \mathrm{Br}_{2} \cdot 3 \mathrm{H}_{2} \mathrm{O} \\
& \mathrm{Zn}(\mathrm{TT} 9)_{2} \mathrm{Br}_{2} \cdot \mathrm{H}_{2} \mathrm{O}
\end{aligned}
$$

(b)

Figure 2

(a) Chemical diagram of the triazolotriazole ligand TT9 discussed in this article (the $2 \mathrm{H}$-tautomer is shown). (b) The chemical formulae of the complexes studied in this article. 
respect to the $2 \mathrm{H}$-tautomer, in the most favourable case) (Centore et al., 2015), the calculated energy of the $3 H$ tautomer, in some cases, is greater than for the $2 \mathrm{H}$-tautomer by only $1 \mathrm{kcal} \mathrm{mol}^{-1}$ or less (Centore et al., 2015). Despite this, the $3 \mathrm{H}$-tautomer should be considered elusive, because it has not yet been observed in the solid state for any of the pure triazolotriazole compounds studied so far.

We have recently described a new versatile nitrogenrich triazolotriazole ligand, 4-methyl-7-(pyrazin-2-yl)- $2 \mathrm{H}, 3 \mathrm{H}$ $[1,2,4]$ triazolo[3,2-c][1,2,4]triazole, henceforth TT9 (Fig. 2a), whose tautomeric behaviour is further enriched by the possibility of metal coordination (Parisi et al., 2020).

In particular, while crystallization of the pure neutral ligand yielded the most stable $2 \mathrm{H}$-tautomer, as expected, crystallization of neutral TT9 in the presence of $\mathrm{Zn}^{\mathrm{II}}$ and $\mathrm{Cu}^{\mathrm{II}}$ salts yielded metal complexes of the neutral ligand, with a $1: 1$ metal-to-ligand ratio, in which the $3 H$-tautomer is present (Parisi et al., 2020). In order to further confirm metal complexation as a way of stabilizing the elusive $3 \mathrm{H}$-tautomer, we report, in this article, structural data for the $\mathrm{Zn}^{\mathrm{II}}$ and $\mathrm{Cu}^{\mathrm{II}}$ complexes of TT9, with a 1:2 metal-to-ligand ratio (Fig. $2 b$ ).

\section{Experimental}

All reagents were of analytical grade and were used without further purification.

\subsection{Synthesis and crystallization}

The synthesis of the TT9 ligand was performed according to the procedure already described by us (Parisi et al., 2020). Prismatic green crystals of the complex $\mathrm{Cu}(\mathrm{TT} 9)_{2} \mathrm{Br}_{2} \cdot 3 \mathrm{H}_{2} \mathrm{O}$, henceforth complex $\mathbf{1}$, were grown in $2 \mathrm{~d}$ by slow evaporation of a clear 50:50 $(\mathrm{v} / \mathrm{v})$ water-ethanol solution containing a 1:2 molar ratio of $\mathrm{CuBr}_{2}$ dihydrate $\left(13.0 \mathrm{mg}, 7.63 \times 10^{-5} \mathrm{~mol}\right)$ and TT9 $\left(31.0 \mathrm{mg}, 1.56 \times 10^{-4} \mathrm{~mol}\right)$ at room temperature in a quantitative yield $(53.0 \mathrm{mg})$. Prismatic brown crystals of the complex $\mathrm{Zn}(\mathrm{TT} 9)_{2} \mathrm{Br}_{2} \cdot \mathrm{H}_{2} \mathrm{O}$, henceforth complex 2, were grown in a week by slow evaporation of a 50:50 $(\mathrm{v} / \mathrm{v})$ waterethanol solution containing a 1:2 molar ratio of $\mathrm{ZnBr}_{2}(14 \mathrm{mg}$,

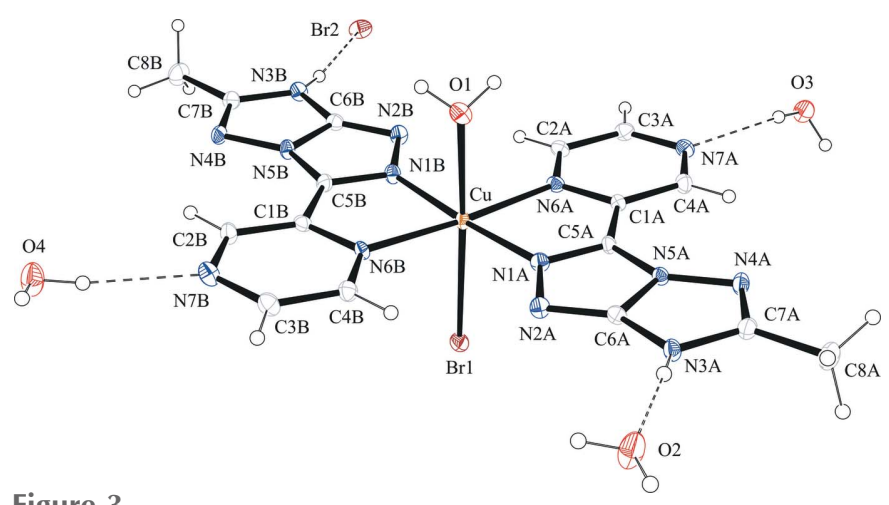

Figure 3

The molecular structure of complex 1, with displacement ellipsoids drawn at the $30 \%$ probability level. Selected hydrogen bonds are indicated by dashed lines.
Table 2

Hydrogen-bond geometry $\left(\AA,^{\circ}\right)$ for $\mathbf{1}$.

\begin{tabular}{lllll}
\hline$D-\mathrm{H} \cdots A$ & $D-\mathrm{H}$ & $\mathrm{H} \cdots A$ & $D \cdots A$ & $D-\mathrm{H} \cdots A$ \\
\hline $\mathrm{C} 2 A-\mathrm{H} 2 A \cdots \mathrm{N} 2 B$ & 0.95 & 2.38 & $3.137(8)$ & 137 \\
$\mathrm{C} 3 A-\mathrm{H} 3 A \cdots \mathrm{Br} 1^{\mathrm{i}}$ & 0.95 & 3.11 & $4.058(6)$ & 173 \\
$\mathrm{~N} 3 A-\mathrm{H} 3 \mathrm{~N} A \cdots \mathrm{O} 2$ & $0.86(3)$ & $1.82(3)$ & $2.657(7)$ & $165(7)$ \\
$\mathrm{C} 4 B-\mathrm{H} 4 B \cdots \mathrm{N} 2 A$ & 0.95 & 2.52 & $3.276(8)$ & 136 \\
$\mathrm{C} 4 B-\mathrm{H} 4 B \cdots \mathrm{Br}{ }^{\text {ii }}$ & 0.95 & 3.09 & $3.848(6)$ & 138 \\
$\mathrm{~N} 3 B-\mathrm{H} 3 \mathrm{~N} B \cdots \mathrm{Br} 2$ & $0.85(3)$ & $2.34(3)$ & $3.190(5)$ & $173(7)$ \\
$\mathrm{O} 1-\mathrm{H} 1 W \cdots \mathrm{Br} 1^{\mathrm{iii}}$ & $0.83(3)$ & $2.42(3)$ & $3.229(5)$ & $166(6)$ \\
$\mathrm{O} 1-\mathrm{H} 2 W \cdots \mathrm{Br} 2^{\mathrm{ii}}$ & $0.83(3)$ & $2.49(3)$ & $3.301(5)$ & $168(8)$ \\
$\mathrm{O} 2-\mathrm{H} 3 W \cdots \mathrm{Br} 1^{\text {ii }}$ & $0.82(3)$ & $2.43(3)$ & $3.237(5)$ & $173(9)$ \\
$\mathrm{O} 2-\mathrm{H} 4 W \cdots \mathrm{O} 3^{\text {iv }}$ & $0.81(3)$ & $1.89(4)$ & $2.691(7)$ & $167(10)$ \\
$\mathrm{O} 3-\mathrm{H} 5 W \cdots \mathrm{O} 4^{\text {v }}$ & $0.79(3)$ & $2.08(6)$ & $2.746(7)$ & $141(8)$ \\
$\mathrm{O} 3-\mathrm{H} 6 W \cdots \mathrm{N} 7 A$ & $0.80(3)$ & $2.17(4)$ & $2.931(7)$ & $159(9)$ \\
$\mathrm{O} 4-\mathrm{H} 7 W \cdots \mathrm{N} 7 B$ & $0.83(3)$ & $2.12(3)$ & $2.939(7)$ & $171(9)$ \\
$\mathrm{O} 4-\mathrm{H} 8 W \cdots \mathrm{Br} 2^{\text {iv }}$ & $0.81(3)$ & $2.56(6)$ & $3.272(6)$ & $147(8)$ \\
\hline
\end{tabular}

Symmetry codes: (i) $x, y-1, z$; (ii) $x+\frac{1}{2}, y+\frac{1}{2}, z$; (iii) $x+\frac{1}{2}, y-\frac{1}{2}, z$; (iv) $x+\frac{1}{2}, y+\frac{3}{2}, z$; (v) $x,-y+1, z+\frac{1}{2}$.

$0.1 \mathrm{mmol})$ and TT9 $(40 \mathrm{mg}, 0.2 \mathrm{mmol})$ at room temperature in a $75 \%$ yield $(48 \mathrm{mg})$.

\subsection{Refinement}

Crystal data, data collection and structure refinement details are summarized in Table $1 . \mathrm{H}$ atoms bonded to $\mathrm{C}$ atoms were generated stereochemically and refined by the riding model. After having placed C-bound $\mathrm{H}$ atoms, those bonded to $\mathrm{O}$ and $\mathrm{N}$ atoms, that are essential in the identification of tautomers, were clearly found in difference Fourier maps as the first maxima and, in some cases, their coordinates were refined. For all $\mathrm{H}$ atoms, $U_{\text {iso }}(\mathrm{H})=1.2 U_{\text {eq }}$ of the carrier atom was assumed (1.5 in the case of methyl groups). The structure of complex $\mathbf{1}$, in the noncentrosymmetric space group $C c$, was refined as a two-component inversion twin.

\section{Results and discussion}

The X-ray molecular structure of complex $\mathbf{1}$ is shown in Fig. 3. Two $3 H$-tautomeric $s$-cis TT9 neutral ligands are coordinated to copper(II) as bidentate chelates (N1 and N6) in a squareplanar arrangement, with the formation of pentatomic chelate rings. The four metal-to-ligand distances in the equatorial plane show a clear and significant asymmetry. In fact, the bond lengths with $\mathrm{N}$-pyrazinic donors $[\mathrm{Cu}-\mathrm{N} 6 A=2.117$ (5) and $\mathrm{Cu}-\mathrm{N} 6 B=2.125(5) \AA]$ are longer than with $\mathrm{N}$-triazole donors $[\mathrm{Cu}-\mathrm{N} 1 A=1.953(5)$ and $\mathrm{Cu}-\mathrm{N} 1 B=1.951(5) \AA]$. This presumably reflects the fact that the pyrazine $\mathrm{N}$ atom is a poorer donor. One water molecule and one bromide ion complete the coordination environment of $\mathrm{Cu}^{\mathrm{II}}$, with transelongated bond lengths of $\mathrm{Cu}-\mathrm{O}=2.410$ (5) and $\mathrm{Cu}-\mathrm{Br}=$ 2.7466 (12) $\AA$. The coordination geometry can be described as octahedral with tetragonal distortion. The observed coordination geometry is typical of $\mathrm{Cu}^{\mathrm{II}}$ and can be related to JahnTeller distortions (Cotton et al., 1999).

The selection of the $3 \mathrm{H}$-tautomer, in complex $\mathbf{1}$, is probably related to the strong preference of $\mathrm{Cu}^{\mathrm{II}}$ for square-planar coordination with $\mathrm{N}$-donor atoms. The formation of penta- 


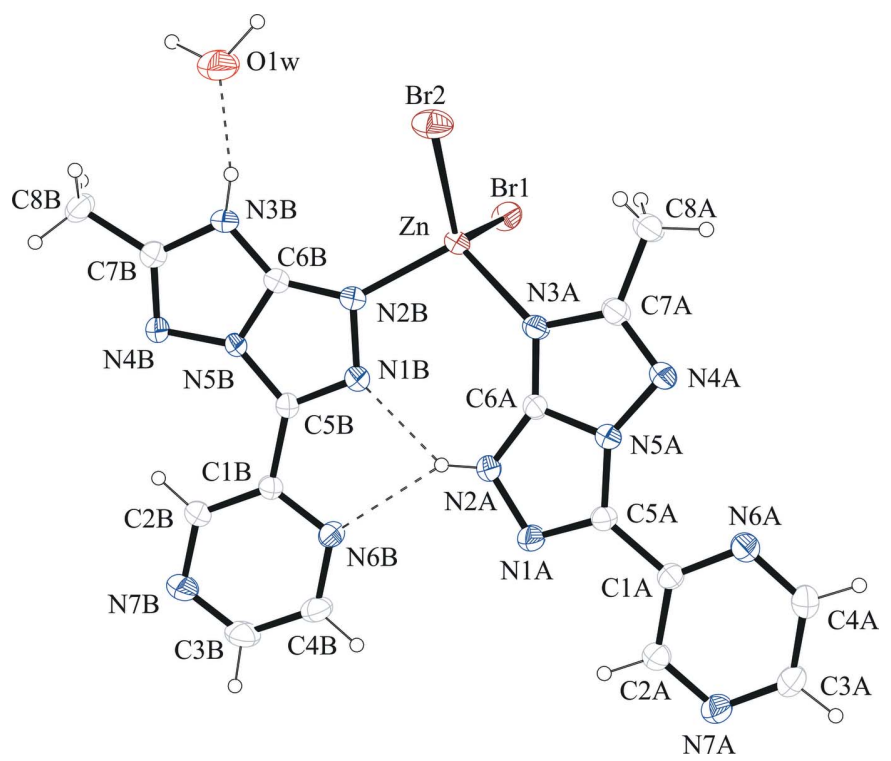

Figure 4

The molecular structure of complex $\mathbf{2}$, with displacement ellipsoids drawn at the $30 \%$ probability level. Selected hydrogen bonds are indicated by dashed lines.

tomic (N1 and N6) chelate rings drives the selection of the $s$-cis conformer and the switching of the proton from $\mathrm{N} 2$ to N3. In fact, in the observed molecular structure, there are close intramolecular contacts (weak hydrogen bonds) $\mathrm{C} 2 A-$ $\mathrm{H} 2 A \cdots \mathrm{N} 2 B$ and $\mathrm{C} 4 B-\mathrm{H} 4 B \cdots \mathrm{N} 2 A$ (Table 2). Three uncoordinated water molecules and a bromide ion are also present in the crystallographically independent unit. They are involved in strong hydrogen bonds with the $\mathrm{N}-\mathrm{H}$ donors and $\mathrm{N}$-atom acceptors present on the rim of the coordinated ligands (Table 2).

The X-ray molecular structure of complex 2, shown in Fig. 4, has completely different features. The coordination around $\mathrm{Zn}^{\mathrm{II}}$ is basically tetrahedral and is accomplished through two bromide ions $[\mathrm{Zn}-\mathrm{Br} 1 \quad 2.4017(11)$ and $\mathrm{Zn}-\mathrm{Br} 2$<smiles>Cc1nc2[nH]nc(-c3cnccn3)n2n1</smiles><smiles>Cc1nc2[nH]nc(-c3cnccn3)n2n1</smiles>

$2 \mathrm{H} \mathrm{s}$-cis, $\mathrm{E}=0 \mathrm{kcal} / \mathrm{mol}$ $2 \mathrm{H}$ s-trans, $\mathrm{E}=0.1 \mathrm{kcal} / \mathrm{mol}$<smiles>Cc1nn2c(-c3cnccn3)nnc2[nH]1</smiles><smiles>Cc1nn2c(-c3cnccn3)nnc2[nH]1</smiles>

$3 H \mathrm{~s}$-cis, $\mathrm{E}=1.9 \mathrm{kcal} / \mathrm{mol} \quad 3 \mathrm{H} \mathrm{s}$-trans, $\mathrm{E}=1.4 \mathrm{kcal} / \mathrm{mol}$

Figure 5

Low-energy tautomers/conformers of TT9. Relative energies are taken from Parisi et al. (2020) and are evaluated in water as the continuum polarizable medium.
Table 3

Hydrogen-bond geometry $\left(\AA,^{\circ}\right)$ for 2 .

\begin{tabular}{lllll}
\hline$D-\mathrm{H} \cdots A$ & $D-\mathrm{H}$ & $\mathrm{H} \cdots A$ & $D \cdots A$ & $D-\mathrm{H} \cdots A$ \\
\hline $\mathrm{C} 2 A-\mathrm{H} 2 A \cdots \mathrm{Br} 1^{\mathrm{i}}$ & 0.93 & 3.13 & $3.995(6)$ & 155 \\
$\mathrm{~N} 2 A-\mathrm{H} 2 \mathrm{~N} A \cdots \mathrm{N} 1 B$ & $0.84(2)$ & $2.09(4)$ & $2.786(6)$ & $139(5)$ \\
$\mathrm{N} 2 A-\mathrm{H} 2 \mathrm{~N} A \cdots \mathrm{N} 6 B$ & $0.84(2)$ & $2.39(4)$ & $3.126(6)$ & $145(5)$ \\
$\mathrm{C} 2 B-\mathrm{H} 2 B \cdots \mathrm{N} 4 B$ & 0.93 & 2.62 & $3.276(7)$ & 129 \\
$\mathrm{C} 4 B-\mathrm{H} 4 B \cdots \mathrm{Br} 1^{\mathrm{i}}$ & 0.93 & 2.86 & $3.791(6)$ & 176 \\
$\mathrm{~N} 3 B-\mathrm{H} 3 \mathrm{~N} B \cdots \mathrm{O} 1 W$ & $0.85(2)$ & $1.85(3)$ & $2.669(6)$ & $163(6)$ \\
$\mathrm{O} 1 W-\mathrm{H} 1 W \cdots \mathrm{Br} 1^{i i}$ & 1.03 & 2.39 & $3.375(6)$ & 162 \\
$\mathrm{O} 1 W-\mathrm{H} 2 W \cdots \mathrm{N} 7 A^{\mathrm{iii}}$ & 0.98 & 2.03 & $2.901(7)$ & 148 \\
\hline
\end{tabular}

Symmetry codes: (i) $-x+1,-y,-z+1$; (ii) $-x,-y+1,-z+1$; (iii) $x-1, y+1, z$.

2.3581 (9) $\AA$ ] and two TT9 ligands acting in a monodentate manner $[\mathrm{Zn}-\mathrm{N} 3 A=2.059$ (4) and $\mathrm{Zn}-\mathrm{N} 2 B=2.018$ (4) $\mathrm{A}]$. The two TT9 ligands are present in different tautomer/ conformers. Ligand $A$ is $2 H$-tautomeric s-trans, whereas ligand $B$ is $3 H$-tautomeric $s$-cis. In the complex, the two ligands are hydrogen bonded to each other through a strong bifurcated hydrogen bond, $\mathrm{N} 2 A-\mathrm{H} 2 \mathrm{~N} A \cdots \mathrm{N} 1 B$ and $\mathrm{N} 2 A-$ $\mathrm{H} 2 \mathrm{~N} A \cdots \mathrm{N} 6 B$ (Table 3). Examples of complexes in which two different tautomeric forms of the same ligand are coordinated to the same metal centre are rare for anionic ligands (Sutradhar et al., 2016), and, to the best of our knowledge, not previously documented for neutral ligands.

A deep inspection of the structure of complex 2 can suggest some basic points for the rational design of such mixedtautomeric-ligand complexes. The two tautomeric forms should have similar ligand-donor capability and similar energy, in such a way that both are present in solution in similar amounts. They should possess complementary functional groups to form stable adducts by secondary interactions (e.g. hydrogen bonds), with a strong preference for mixed adducts. Finally, the mixed hydrogen-bonded adduct should be featured with a pocket, suitable for the dimensions and presence of donor atoms, to bind a metal ion. The fulfilment of all these issues may account for the rarity of the phenomenon.

The tautomeric/conformational variability of the TT9 ligand, which possesses four different tautomers/conformers within a narrow energy range $\Delta E<2 \mathrm{kcal} \mathrm{mol}^{-1}$ (Parisi et al., 2020), makes TT9 a reliable candidate for this target. In fact, we have considered the formation of hydrogen-bonded dimers for the four lowest-energy tautomers/conformers of TT9, shown in Fig. 5 (Parisi et al., 2020). Our analysis showed that, of the ten possible combinations, only in four cases can hydrogen-bonded dimers be formed, and they are shown in Fig. 6. In all the dimers, a strong (bifurcated) hydrogen bond is present between the $\mathrm{N}-\mathrm{H}$ donor of a tautomer and the two $s$-cis $\mathrm{N}$-acceptor atoms of the other. The hydrogen-bonded dimers also show a pocket with two N-donor atoms that could host a metal ion, for instance, in tetrahedral coordination geometry. Of the four possible dimers, two are homotautomeric and contain the higher-energy $3 \mathrm{H}$-tautomer, while the other two are heterotautomeric and contain both $2 \mathrm{H}$ - and $3 H$-tautomers. Evidently, the two latter, $2 \mathrm{H} \mathrm{s}$-trans $/ 3 \mathrm{H} s$-cis and $2 \mathrm{Hs}$-cis/3H s-cis, are energetically more feasible and their energies should be very close, within $0.1 \mathrm{kcal} \mathrm{mol}^{-1}$. 
Thus, our analysis confirms that the formation of mixedtautomer complexes can be expected with TT9.

\subsection{Supramolecular features}

The crystal packing of both complexes is basically driven by the formation of a network of strong hydrogen bonds involving $\mathrm{N}-\mathrm{H}$ donors and $\mathrm{N}$-atom acceptors of the ligand molecules, the bromide ions and the water molecules (see Tables 2 and 3 ).

The equatorial plane of complex $\mathbf{1}$ is neither perpendicular nor parallel to the unique $b$ axis. Therefore, in the crystal, molecules with two different orientations are present. The most remarkable supramolecular architecture is represented by hydrogen-bonded chains running along $a-b$ and $a+b$ (Fig. 7); these directions are equivalent by symmetry in the monoclinic system through the $c$-glide operation perpendicular to the unique $b$ axis. The chains are formed by hydrogen bonding between water donors and bromide acceptors axially coordinated to the metal in consecutive complex molecules along the chains. Different chains are held together laterally by hydrogen bonds involving noncoordinated water molecules.

In complex $\mathbf{2}$, an intramolecular bifurcated hydrogenbonding interaction is present between the $\mathrm{N} 2 A-\mathrm{H} 2 \mathrm{~N} A$ donor group and the $\mathrm{N} 1 B$ and $\mathrm{N} 6 B$ acceptors of the two coordinated ligands. The most remarkable supramolecular architecture is represented by nearly planar hydrogen-bonded ribbons of molecules, running along $a-b$. The ribbons contain<smiles></smiles>

$2 H$ s-trans/3H s-cis

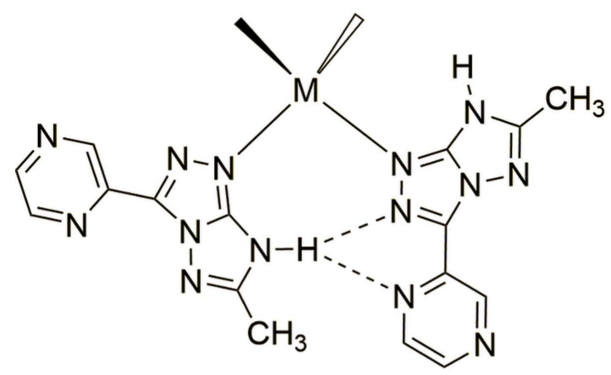

Figure 6

$3 \mathrm{H}$ s-trans/3H s-cis

Hydrogen-bonded dimers of neutral TT9. The presence of a metal atom $M$ in a tetrahedral coordination is also outlined. Highlighted in blue is the dimer found in complex 2 .

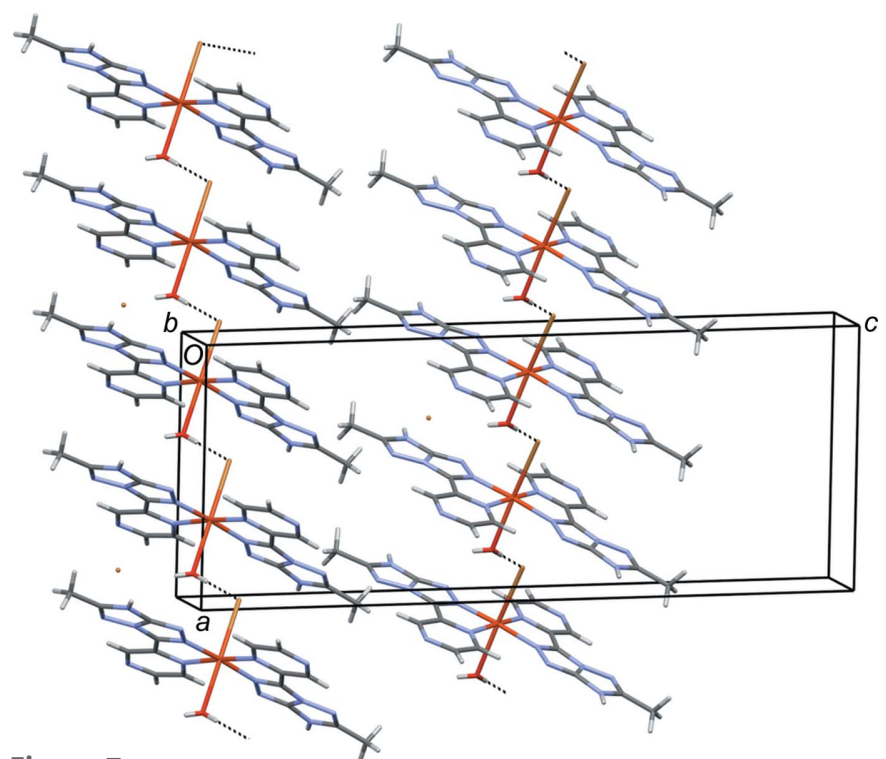

Figure 7

Partial crystal packing of complex 1. Selected hydrogen bonds are indicated by dashed lines.

$2 H$-tautomeric s-trans and $3 H$-tautomeric $s$-cis ligand molecules intercalated by water molecules (Fig. 8).

\section{Conclusions}

Nitrogen-rich ligand TT9, i.e. 4-methyl-7-(pyrazin-2-yl)-2H$[1,2,4]$ triazolo[3,2-c] $[1,2,4]$ triazole, has a low-energy $3 H$-tauto-

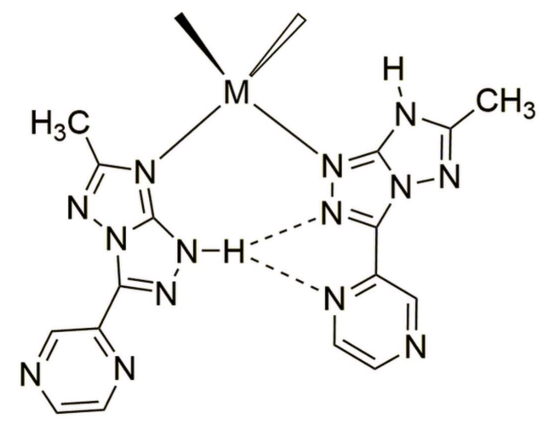

$2 H$ s-cis/3H s-cis

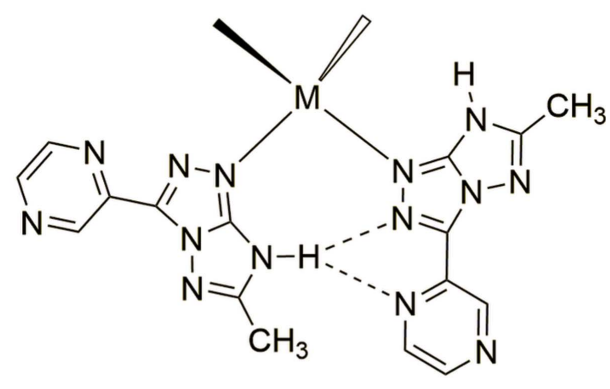

$3 H \mathrm{~s}-\mathrm{cis} / 3 \mathrm{H} \mathrm{s}$-cis 


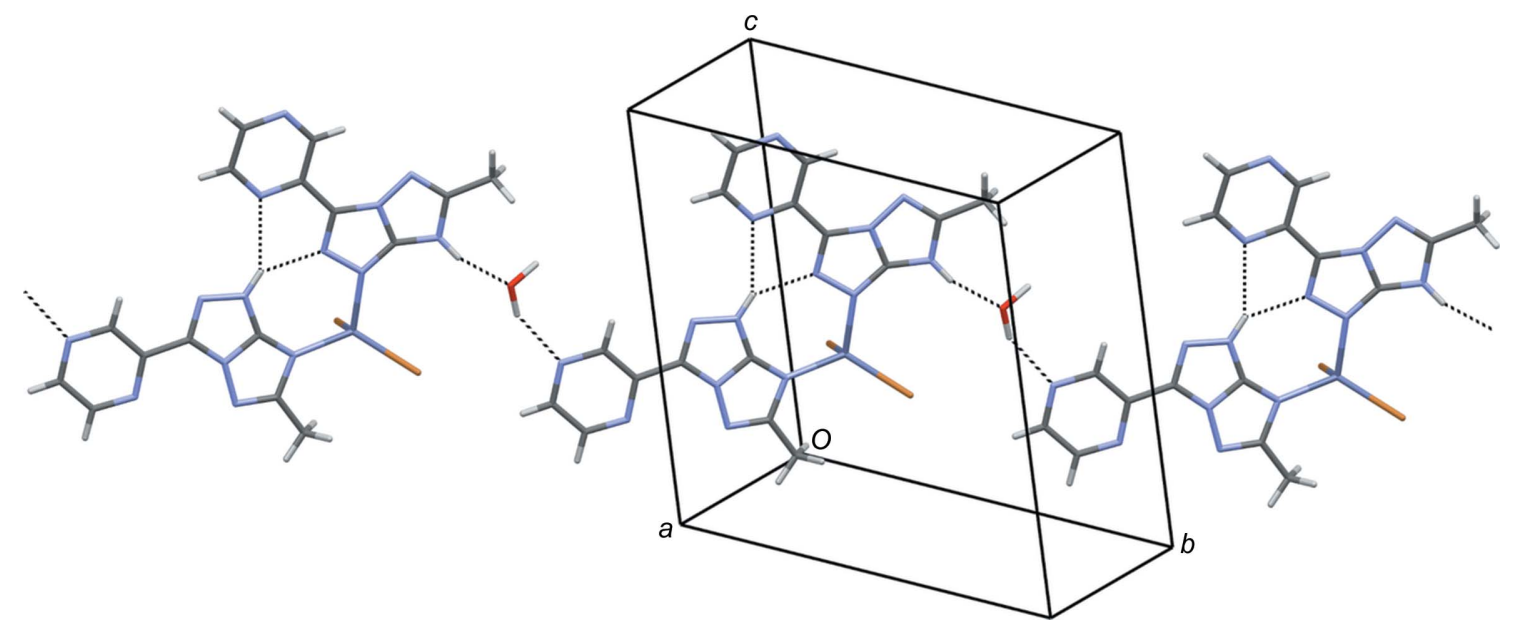

Figure 8

Partial crystal packing of complex 2. Selected hydrogen bonds are indicated by dashed lines.

mer. While crystallization of pure TT9 from different solvents only affords the most stable $2 \mathrm{H}$-tautomer, we have proven that metal coordination allows selection of the elusive $3 \mathrm{H}$ tautomer. Depending on the stoichiometry of the complex, and on the metal, complexes with only the $3 H$-tautomeric ligand (homotautomeric), or complexes with mixed-tautomer ligands, i.e. both $2 H$ and $3 H$ (heterotautomeric), can be obtained.

Steering and selectively controlling the formation of the different energetically feasible tautomers of a given compound, depending on the physico-chemical environment, is of practical and theoretical relevance. In fact, different tautomers generally interact differently with the same substrate and show different properties: in an analogy with language, they have different 'meaning'. After all, chemistry can be considered a language: atoms are its letters and molecules its words. Continuing with this analogy, tautomers, and more generally isomers, would correspond to the anagrams of ordinary language. In this analogy, the heterotautomeric complex 2 would correspond to a sentence containing two anagrams of the same word, e.g. 'she married an admirer'.

This is uncommon also in ordinary language.

\section{Acknowledgements}

The authors would like to acknowledge the contribution of the COST Action CA17120-Chemobrionics. Thanks are also due to the Centro Regionale di Competenza NTAP of Regione Campania (Italy) for the X-ray facility.

\section{References}

Altomare, A., Burla, M. C., Camalli, M., Cascarano, G. L., Giacovazzo, C., Guagliardi, A., Moliterni, A. G. G., Polidori, G. \& Spagna, R. (1999). J. Appl. Cryst. 32, 115-119.

Ambrosanio, P., Centore, R., Concilio, S., Panunzi, B., Sirigu, A. \& Tirelli, N. (1999). Polymer, 40, 4923-4928.

Borbone, F., Caruso, U., Centore, R., DeMaria, A., Fort, A., Fusco, M., Panunzi, B., Roviello, A. \& Tuzi, A. (2004). Eur. J. Inorg. Chem. 2004, 2467-2476.
Bruker (2001). SADABS. Bruker AXS Inc., Madison, Wisconsin, USA.

Bussetti, G., Campione, M., Riva, M., Picone, A., Raimondo, L., Ferraro, L., Hogan, C., Palummo, M., Brambilla, A., Finazzi, M., Duò, L., Sassella, A. \& Ciccacci, F. (2014). Adv. Funct. Mater. 24, 958-963.

Centore, R., Concilio, S., Panunzi, B., Sirigu, A. \& Tirelli, N. (1999). J. Polym. Sci. A Polym. Chem. 37, 603-608.

Centore, R., Fusco, S., Capobianco, A., Piccialli, V., Zaccaria, S. \& Peluso, A. (2013). Eur. J. Org. Chem. 2013, 3721-3728.

Centore, R., Manfredi, C., Capobianco, A., Volino, S., Ferrara, M. V., Carella, A., Fusco, S. \& Peluso, A. (2017). J. Org. Chem. 82, 51555161.

Centore, R., Manfredi, C., Fusco, S., Maglione, C., Carella, A., Capobianco, A., Peluso, A., Colonna, D. \& Di Carlo, A. (2015). J. Mol. Struct. 1093, 119-124.

Centore, R., Panunzi, B., Roviello, A., Sirigu, A. \& Villano, P. (1996). J. Polym. Sci. A Polym. Chem. 34, 3203-3211.

Cotton, F. A., Wilkinson, G., Murillo, C. A. \& Bochmann, M. (1999). In Advanced Inorganic Chemistry, 6th ed. New York: John Wiley \& Sons Inc.

Deneva, V., Dobrikov, G., Crochet, A., Nedeltcheva, D., Fromm, K. M. \& Antonov, L. (2019). J. Org. Chem. 15, 1898-1906.

Duisenberg, A. J. M., Hooft, R. W. W., Schreurs, A. M. M. \& Kroon, J. (2000). J. Appl. Cryst. 33, 893-898.

Duisenberg, A. J. M., Kroon-Batenburg, L. M. J. \& Schreurs, A. M. M. (2003). J. Appl. Cryst. 36, 220-229.

Farrugia, L. J. (2012). J. Appl. Cryst. 45, 849-854.

Fusco, S., Parisi, E., Carella, A., Capobianco, A., Peluso, A., Manfredi, C., Borbone, F. \& Centore, R. (2018). Cryst. Growth Des. 18, 6293-6301.

Goodman, M. (1995). Nature, 378, 237-238.

Horiuchi, S., Kobayashi, K., Kumai, R. \& Ishibashi, S. (2017). Nat. Commun. 8, 14426.

Macrae, C. F., Sovago, I., Cottrell, S. J., Galek, P. T. A., McCabe, P., Pidcock, E., Platings, M., Shields, G. P., Stevens, J. S., Towler, M. \& Wood, P. A. (2020). J. Appl. Cryst. 53, 226-235.

Martin, C. Y. (2009). J. Comput. Aided Mol. Des. 23, 693-704.

McNaught, A. D. \& Wilkinson, A. (1997). In IUPAC Compendium of Chemical Terminology, 2nd ed. Oxford: Blackwell Science.

Nonius (1999). COLLECT. Nonius BV, Delft, The Netherlands.

Parisi, E., Capasso, D., Capobianco, A., Peluso, A., Di Gaetano, S., Fusco, S., Manfredi, C., Mozzillo, R., Pinto, G. \& Centore, R. (2020). Dalton Trans. 49, 14452-14462.

Sheldrick, G. M. (2015). Acta Cryst. C71, 3-8. 
Sutradhar, M., Alegria, E. C. B. A., Mahmudov, T., Guedes da Silva, F. C. \& Pombeiro, A. J. L. (2016). RSC Adv., 6, 8079-8088.

Takjoo, R. \& Centore, R. (2013). J. Mol. Struct. 1031, 180-185.

Taylor, P. J., Van der Zwan, G. \& Antonov, L. (2014). In Tautomerism: Methods and Theories. Weinheim: Wiley-VCH Verlag GmbH \& Co.
Todorov, A. R., Nieger, M. \& Helaja, J. (2012). Chem. Eur. J. 18, 7269-7277.

Wang, W., Hellinga, W. \& Beese, L. S. (2011). Proc. Natl Acad. Sci. USA, 108, 17644-17648.

Watson, J. D. \& Crick, F. H. (1953a). Nature, 171, 737-738.

Watson, J. D. \& Crick, F. H. (1953b). Nature, 171, 964-967. 


\section{supporting information}

Acta Cryst. (2021). C77, 395-401 [https://doi.org/10.1107/S2053229621006203]

\section{Stabilization of an elusive tautomer by metal coordination}

\section{Emmanuele Parisi and Roberto Centore}

\section{Computing details}

For both structures, data collection: COLLECT (Nonius, 1999); cell refinement: DIRAX/LSQ (Duisenberg et al., 2000); data reduction: EVALCCD (Duisenberg et al., 2003); program(s) used to solve structure: SIR97 (Altomare et al., 1999); program(s) used to refine structure: SHELXL2016 (Sheldrick, 2015); molecular graphics: ORTEP-3 for Windows (Farrugia, 2012) and Mercury (Macrae et al., 2020); software used to prepare material for publication: WinGX (Farrugia, 2012).

Aquabromidobis[4-methyl-7-(pyrazin-2-yl)-3H-[1,2,4] triazolo[3,2-c][1,2,4]triazole]copper(II) bromide trihydrate (1)

\section{Crystal data}

$\left[\mathrm{CuBr}\left(\mathrm{C}_{8} \mathrm{H}_{7} \mathrm{~N}_{7}\right)_{2}\left(\mathrm{H}_{2} \mathrm{O}\right)\right] \mathrm{Br} \cdot 3 \mathrm{H}_{2} \mathrm{O}$

$M_{r}=697.83$

Monoclinic, $C c$ $a=11.062(4) \AA$

$b=8.369(3) \AA$

$c=27.406(6) \AA$

$\beta=92.44(3)^{\circ}$

$V=2534.9(14) \AA^{3}$

$Z=4$

\section{Data collection}

Bruker-Nonius KappaCCD diffractometer

Radiation source: normal-focus sealed tube Graphite monochromator

Detector resolution: 9 pixels $\mathrm{mm}^{-1}$

CCD rotation images, thick slices scans

Absorption correction: multi-scan (SADABS; Bruker, 2001)

$T_{\text {min }}=0.250, T_{\max }=0.478$

Refinement

Refinement on $F^{2}$

Least-squares matrix: full

$R\left[F^{2}>2 \sigma\left(F^{2}\right)\right]=0.033$

$w R\left(F^{2}\right)=0.081$

$S=1.04$

4870 reflections

367 parameters

13 restraints
$F(000)=1388$

$D_{\mathrm{x}}=1.829 \mathrm{Mg} \mathrm{m}^{-3}$

Mo $K \alpha$ radiation, $\lambda=0.71073 \AA$

Cell parameters from 320 reflections

$\theta=3.3-28.5^{\circ}$

$\mu=4.07 \mathrm{~mm}^{-1}$

$T=173 \mathrm{~K}$

Prismatic, green

$0.50 \times 0.20 \times 0.20 \mathrm{~mm}$

8682 measured reflections

4870 independent reflections

4563 reflections with $I>2 \sigma(I)$

$R_{\text {int }}=0.036$

$\theta_{\max }=27.6^{\circ}, \theta_{\min }=3.1^{\circ}$

$h=-13 \rightarrow 14$

$k=-10 \rightarrow 10$

$l=-35 \rightarrow 35$

Primary atom site location: structure-invariant direct methods

Secondary atom site location: difference Fourier map

Hydrogen site location: mixed

$\mathrm{H}$ atoms treated by a mixture of independent and constrained refinement 
$w=1 /\left[\sigma^{2}\left(F_{\mathrm{o}}^{2}\right)+(0.0413 P)^{2}+2.1083 P\right]$

where $P=\left(F_{\mathrm{o}}^{2}+2 F_{\mathrm{c}}{ }^{2}\right) / 3$

$(\Delta / \sigma)_{\max }<0.001$

$\Delta \rho_{\max }=0.60 \mathrm{e} \AA^{-3}$
$\Delta \rho_{\min }=-0.86$ e $\AA^{-3}$

Absolute structure: Refined as an inversion twin.

Absolute structure parameter: 0.502 (12)

\section{Special details}

Geometry. All esds (except the esd in the dihedral angle between two 1.s. planes) are estimated using the full covariance matrix. The cell esds are taken into account individually in the estimation of esds in distances, angles and torsion angles; correlations between esds in cell parameters are only used when they are defined by crystal symmetry. An approximate (isotropic) treatment of cell esds is used for estimating esds involving l.s. planes.

Refinement. Refined as a 2-component inversion twin.

Data were collected on a Bruker-Nonius KappaCCD diffractometer equipped with Oxford Cryostream 700 apparatus. The structures were solved by direct methods and refined by the full matrix least squares method with anisotropic displacement parameters for non-H atoms.

Fractional atomic coordinates and isotropic or equivalent isotropic displacement parameters $\left(\AA^{2}\right)$

\begin{tabular}{|c|c|c|c|c|}
\hline & $x$ & $y$ & $z$ & $U_{\text {iso }} * / U_{\text {eq }}$ \\
\hline $\mathrm{C} 1 \mathrm{~A}$ & $0.2108(5)$ & $0.3482(7)$ & $0.1008(2)$ & $0.0171(11)$ \\
\hline $\mathrm{C} 2 \mathrm{~A}$ & $0.0988(5)$ & $0.1833(7)$ & $0.0494(2)$ & $0.0203(12)$ \\
\hline $\mathrm{H} 2 \mathrm{~A}$ & 0.055550 & 0.163375 & 0.019347 & $0.024 *$ \\
\hline $\mathrm{C} 3 \mathrm{~A}$ & $0.1070(6)$ & $0.0641(7)$ & $0.0854(2)$ & $0.0233(12)$ \\
\hline $\mathrm{H} 3 \mathrm{~A}$ & 0.070052 & -0.036484 & 0.078759 & $0.028 *$ \\
\hline $\mathrm{C} 4 \mathrm{~A}$ & $0.2180(6)$ & $0.2281(7)$ & $0.1359(2)$ & $0.0218(12)$ \\
\hline $\mathrm{H} 4 \mathrm{~A}$ & 0.261924 & 0.247106 & 0.165862 & $0.026 *$ \\
\hline C5A & $0.2645(5)$ & $0.5042(7)$ & $0.1058(2)$ & $0.0164(11)$ \\
\hline C6A & $0.3529(5)$ & $0.7240(7)$ & $0.1263(2)$ & $0.0177(11)$ \\
\hline C7A & $0.4323(6)$ & $0.6809(8)$ & $0.1994(2)$ & $0.0227(12)$ \\
\hline $\mathrm{C} 8 \mathrm{~A}$ & $0.5012(7)$ & $0.7127(9)$ & $0.2462(2)$ & $0.0322(15)$ \\
\hline $\mathrm{H} 8 \mathrm{~A}$ & 0.448925 & 0.767907 & 0.268770 & $0.048 *$ \\
\hline H8B & 0.571526 & 0.779821 & 0.240018 & $0.048 *$ \\
\hline $\mathrm{H} 8 \mathrm{C}$ & 0.528625 & 0.611249 & 0.260743 & $0.048 *$ \\
\hline N1A & $0.2503(5)$ & $0.6096(6)$ & $0.07033(17)$ & $0.0184(10)$ \\
\hline $\mathrm{N} 2 \mathrm{~A}$ & $0.3066(5)$ & $0.7527(6)$ & $0.08205(18)$ & $0.0195(10)$ \\
\hline N3A & $0.4212(5)$ & $0.7944(6)$ & $0.16306(18)$ & $0.0195(10)$ \\
\hline H3NA & $0.461(6)$ & $0.882(5)$ & $0.162(3)$ & $0.023 *$ \\
\hline $\mathrm{N} 4 \mathrm{~A}$ & $0.3787(5)$ & $0.5451(6)$ & $0.18878(17)$ & $0.0219(10)$ \\
\hline N5A & 0.3309 (4) & $0.5749(6)$ & $0.14234(16)$ & $0.0160(9)$ \\
\hline N6A & 0.1518 (4) & $0.3249(5)$ & $0.05743(18)$ & $0.0180(10)$ \\
\hline N7A & $0.1644(5)$ & $0.0863(6)$ & $0.12843(18)$ & $0.0226(11)$ \\
\hline C1B & $0.1316(5)$ & $0.6931(7)$ & $-0.0809(2)$ & $0.0184(11)$ \\
\hline $\mathrm{C} 2 \mathrm{~B}$ & $0.1366(6)$ & 0.7999 (7) & -0.1188 & $0.0212(12)$ \\
\hline $\mathrm{H} 2 \mathrm{~B}$ & 0.101996 & 0.771551 & -0.149940 & $0.025 *$ \\
\hline C3B & $0.2360(6)$ & $0.9747(8)$ & $-0.0686(2)$ & $0.0252(13)$ \\
\hline H3B & 0.272402 & 1.076143 & -0.062860 & $0.030^{*}$ \\
\hline $\mathrm{C} 4 \mathrm{~B}$ & $0.2340(6)$ & $0.8649(7)$ & -0.0299 & $0.0214(12)$ \\
\hline H4B & 0.270875 & 0.891593 & 0.000938 & $0.026^{*}$ \\
\hline C5B & $0.0785(6)$ & $0.5357(7)$ & $-0.0844(2)$ & $0.0190(12)$ \\
\hline C6B & $-0.0024(5)$ & $0.3091(7)$ & $-0.1041(2)$ & $0.0191(11)$ \\
\hline
\end{tabular}




$\begin{array}{lllll}\text { C7B } & -0.0630(6) & 0.3334(7) & -0.1805(2) & 0.0236(12) \\ \text { C8B } & -0.1181(7) & 0.2884(9) & -0.2287(2) & 0.0335(16) \\ \text { H8D } & -0.123800 & 0.382944 & -0.249744 & 0.050^{*} \\ \text { H8E } & -0.199277 & 0.244961 & -0.224433 & 0.050^{*} \\ \text { H8F } & -0.067814 & 0.207352 & -0.243794 & 0.050^{*} \\ \text { N1B } & 0.0837(4) & 0.4380(6) & -0.04658(18) & 0.0191(10) \\ \text { N2B } & 0.0320(5) & 0.2912(6) & -0.05801(18) & 0.0209(10) \\ \text { N3B } & -0.0583(5) & 0.2296(6) & -0.14155(18) & 0.0214(10) \\ \text { H3NB } & -0.088(6) & 0.137(5) & -0.137(3) & 0.026^{*} \\ \text { N4B } & -0.0137(5) & 0.4731(6) & -0.17061(17) & 0.0206(11) \\ \text { N5B } & 0.0225(4) & 0.4554(6) & -0.12167(17) & 0.0182(10) \\ \text { N6B } & 0.1811(4) & 0.7247(6) & -0.03631(17) & 0.0160(9) \\ \text { N7B } & 0.1890(5) & 0.9421(6) & -0.1129(2) & 0.0267(12) \\ \text { O1 } & 0.3589(5) & 0.4611(5) & -0.01555(18) & 0.0310(10) \\ \text { H1W } & 0.381(8) & 0.372(5) & -0.005(2) & 0.037^{*} \\ \text { H2W } & 0.362(7) & 0.449(8) & -0.0455(10) & 0.037^{*} \\ \text { O2 } & 0.5055(6) & 1.0913(6) & 0.15962(18) & 0.0420(13) \\ \text { H3W } & 0.492(9) & 1.114(11) & 0.1310(13) & 0.050^{*} \\ \text { H4W } & 0.543(8) & 1.161(8) & 0.174(3) & 0.050^{*} \\ \text { O3 } & 0.1544(5) & -0.1814(5) & 0.19710(16) & 0.0298(11) \\ \text { H5W } & 0.136(8) & -0.139(8) & 0.2215(19) & 0.036^{*} \\ \text { H6W } & 0.176(8) & -0.108(7) & 0.181(3) & 0.036^{*} \\ \text { O4 } & 0.1829(6) & 1.1261(6) & -0.20431(19) & 0.0400(13) \\ \text { H7W } & 0.177(9) & 1.080(10) & -0.1778(18) & 0.048^{*} \\ \text { H8W } & 0.229(6) & 1.200(7) & -0.199(3) & 0.048^{*} \\ \text { Br1 } & -0.05312(4) & 0.64638(7) & 0.04411(2) & 0.02344(15) \\ \text { Br2 } & -0.16760(5) & -0.12171(7) & -0.13330(2) & 0.03047(17) \\ \text { Cu } & 0.16163(6) & 0.52924(8) & 0.01225(3) & 0.01672(15) \\ & & & & \end{array}$

Atomic displacement parameters $\left(\AA^{2}\right)$

\begin{tabular}{lllllll}
\hline & $U^{11}$ & $U^{22}$ & $U^{33}$ & $U^{12}$ & $U^{13}$ & $U^{23}$ \\
\hline C1A & $0.019(3)$ & $0.016(3)$ & $0.016(2)$ & $0.003(2)$ & $-0.001(2)$ & $0.001(2)$ \\
C2A & $0.020(3)$ & $0.016(3)$ & $0.025(3)$ & $0.001(2)$ & $0.000(2)$ & $-0.001(2)$ \\
C3A & $0.027(3)$ & $0.018(3)$ & $0.026(3)$ & $-0.003(2)$ & $0.004(2)$ & $-0.001(2)$ \\
C4A & $0.023(3)$ & $0.023(3)$ & $0.019(3)$ & $0.001(2)$ & $0.001(2)$ & $0.005(2)$ \\
C5A & $0.016(3)$ & $0.018(3)$ & $0.014(2)$ & $0.001(2)$ & $-0.004(2)$ & $0.001(2)$ \\
C6A & $0.017(3)$ & $0.015(3)$ & $0.021(3)$ & $-0.001(2)$ & $0.001(2)$ & $0.000(2)$ \\
C7A & $0.022(3)$ & $0.028(3)$ & $0.018(2)$ & $0.003(3)$ & $-0.001(2)$ & $-0.001(2)$ \\
C8A & $0.039(4)$ & $0.034(4)$ & $0.022(3)$ & $-0.002(3)$ & $-0.011(3)$ & $-0.005(3)$ \\
N1A & $0.020(3)$ & $0.015(2)$ & $0.020(2)$ & $-0.0028(19)$ & $-0.001(2)$ & $-0.0004(18)$ \\
N2A & $0.023(3)$ & $0.014(2)$ & $0.021(2)$ & $-0.007(2)$ & $-0.005(2)$ & $-0.0019(19)$ \\
N3A & $0.021(3)$ & $0.019(3)$ & $0.018(2)$ & $0.000(2)$ & $-0.005(2)$ & $0.0017(19)$ \\
N4A & $0.026(3)$ & $0.024(3)$ & $0.015(2)$ & $0.001(2)$ & $-0.004(2)$ & $0.0015(19)$ \\
N5A & $0.018(2)$ & $0.013(2)$ & $0.016(2)$ & $-0.0007(18)$ & $-0.0020(19)$ & $0.0007(17)$ \\
N6A & $0.019(2)$ & $0.015(2)$ & $0.021(2)$ & $0.0009(19)$ & $0.0037(19)$ & $0.0017(18)$ \\
N7A & $0.028(3)$ & $0.015(2)$ & $0.025(2)$ & $0.001(2)$ & $0.001(2)$ & $0.0028(19)$ \\
C1B & $0.016(3)$ & $0.018(3)$ & $0.021(2)$ & $0.004(2)$ & $0.003(2)$ & $0.002(2)$
\end{tabular}




$\begin{array}{lllllll}\text { C2B } & 0.026(3) & 0.019(3) & 0.018(3) & -0.002(2) & -0.004(2) & 0.002(2) \\ \text { C3B } & 0.023(3) & 0.023(3) & 0.029(3) & 0.001(2) & -0.004(3) & 0.002(3) \\ \text { C4B } & 0.021(3) & 0.023(3) & 0.020(3) & 0.001(2) & -0.003(2) & 0.000(2) \\ \text { C5B } & 0.023(3) & 0.017(3) & 0.017(2) & -0.001(2) & 0.003(2) & 0.003(2) \\ \text { C6B } & 0.020(3) & 0.016(3) & 0.022(3) & -0.001(2) & 0.002(2) & 0.001(2) \\ \text { C7B } & 0.025(3) & 0.025(3) & 0.020(3) & 0.003(3) & -0.002(2) & -0.001(2) \\ \text { C8B } & 0.043(4) & 0.033(4) & 0.024(3) & -0.004(3) & -0.005(3) & -0.007(3) \\ \text { N1B } & 0.021(3) & 0.018(2) & 0.018(2) & -0.0035(19) & -0.0010(19) & 0.0033(19) \\ \text { N2B } & 0.025(3) & 0.017(2) & 0.020(2) & -0.005(2) & -0.005(2) & -0.0017(19) \\ \text { N3B } & 0.024(3) & 0.018(3) & 0.022(2) & -0.003(2) & -0.007(2) & -0.0026(19) \\ \text { N4B } & 0.026(3) & 0.022(3) & 0.014(2) & 0.000(2) & -0.004(2) & -0.0011(19) \\ \text { N5B } & 0.019(2) & 0.019(3) & 0.016(2) & -0.0033(19) & -0.0054(19) & 0.0015(18) \\ \text { N6B } & 0.016(2) & 0.015(2) & 0.017(2) & 0.0018(18) & -0.0029(19) & 0.0031(17) \\ \text { N7B } & 0.028(3) & 0.022(3) & 0.030(3) & 0.000(2) & -0.001(2) & 0.006(2) \\ \text { O1 } & 0.038(3) & 0.024(2) & 0.031(2) & 0.008(2) & 0.003(2) & 0.0046(19) \\ \text { O2 } & 0.068(4) & 0.032(3) & 0.026(2) & -0.023(3) & -0.006(3) & 0.002(2) \\ \text { O3 } & 0.041(3) & 0.022(2) & 0.027(3) & -0.006(2) & 0.002(2) & 0.0006(18) \\ \text { O4 } & 0.057(4) & 0.035(3) & 0.026(2) & -0.013(3) & -0.009(3) & 0.005(2) \\ \text { Br1 } & 0.0212(3) & 0.0251(3) & 0.0239(3) & 0.0039(2) & 0.0000(2) & 0.0014(2) \\ \text { Br2 } & 0.0303(4) & 0.0254(3) & 0.0363(3) & -0.0067(3) & 0.0068(3) & -0.0072(3) \\ \text { Cu } & 0.0210(3) & 0.0142(3) & 0.0144(3) & -0.0030(3) & -0.0043(2) & 0.0018(3)\end{array}$

Geometric parameters $\left(A,{ }^{\circ}\right)$

\begin{tabular}{llll}
\hline $\mathrm{C} 1 \mathrm{~A}-\mathrm{N} 6 \mathrm{~A}$ & $1.346(8)$ & $\mathrm{C} 3 \mathrm{~B}-\mathrm{N} 7 \mathrm{~B}$ & $1.329(9)$ \\
$\mathrm{C} 1 \mathrm{~A}-\mathrm{C} 4 \mathrm{~A}$ & $1.391(8)$ & $\mathrm{C} 3 \mathrm{~B}-\mathrm{C} 4 \mathrm{~B}$ & $1.403(9)$ \\
$\mathrm{C} 1 \mathrm{~A}-\mathrm{C} 5 \mathrm{~A}$ & $1.438(8)$ & $\mathrm{C} 3 \mathrm{~B}-\mathrm{H} 3 \mathrm{~B}$ & 0.9500 \\
$\mathrm{C} 2 \mathrm{~A}-\mathrm{N} 6 \mathrm{~A}$ & $1.336(7)$ & $\mathrm{C} 4 \mathrm{~B}-\mathrm{N} 6 \mathrm{~B}$ & $1.320(8)$ \\
$\mathrm{C} 2 \mathrm{~A}-\mathrm{C} 3 \mathrm{~A}$ & $1.404(9)$ & $\mathrm{C} 4 \mathrm{~B}-\mathrm{H} 4 \mathrm{~B}$ & 0.9500 \\
$\mathrm{C} 2 \mathrm{~A}-\mathrm{H} 2 \mathrm{~A}$ & 0.9500 & $\mathrm{C} 5 \mathrm{~B}-\mathrm{N} 1 \mathrm{~B}$ & $1.320(7)$ \\
$\mathrm{C} 3 \mathrm{~A}-\mathrm{N} 7 \mathrm{~A}$ & $1.328(8)$ & $\mathrm{C} 5 \mathrm{~B}-\mathrm{N} 5 \mathrm{~B}$ & $1.350(8)$ \\
$\mathrm{C} 3 \mathrm{~A}-\mathrm{H} 3 \mathrm{~A}$ & 0.9500 & $\mathrm{C} 6 \mathrm{~B}-\mathrm{N} 2 \mathrm{~B}$ & $1.311(8)$ \\
$\mathrm{C} 4 \mathrm{~A}-\mathrm{N} 7 \mathrm{~A}$ & $1.338(8)$ & $\mathrm{C} 6 \mathrm{~B}-\mathrm{N} 5 \mathrm{~B}$ & $1.349(7)$ \\
$\mathrm{C} 4 \mathrm{~A}-\mathrm{H} 4 \mathrm{~A}$ & 0.9500 & $\mathrm{C} 6 \mathrm{~B}-\mathrm{N} 3 \mathrm{~B}$ & $1.352(8)$ \\
$\mathrm{C} 5 \mathrm{~A}-\mathrm{N} 1 \mathrm{~A}$ & $1.317(7)$ & $\mathrm{C} 7 \mathrm{~B}-\mathrm{N} 4 \mathrm{~B}$ & $1.313(8)$ \\
$\mathrm{C} 5 \mathrm{~A}-\mathrm{N} 5 \mathrm{~A}$ & $1.353(7)$ & $\mathrm{C} 7 \mathrm{~B}-\mathrm{N} 3 \mathrm{~B}$ & $1.376(8)$ \\
$\mathrm{C} 6 \mathrm{~A}-\mathrm{N} 2 \mathrm{~A}$ & $1.317(8)$ & $\mathrm{C} 7 \mathrm{~B}-\mathrm{C} 8 \mathrm{~B}$ & $1.480(9)$ \\
$\mathrm{C} 6 \mathrm{~A}-\mathrm{N} 5 \mathrm{~A}$ & $1.349(7)$ & $\mathrm{C} 8 \mathrm{~B}-\mathrm{H} 8 \mathrm{D}$ & 0.9800 \\
$\mathrm{C} 6 \mathrm{~A}-\mathrm{N} 3 \mathrm{~A}$ & $1.368(8)$ & $\mathrm{C} 8 \mathrm{~B}-\mathrm{H} 8 \mathrm{E}$ & 0.9800 \\
$\mathrm{C} 7 \mathrm{~A}-\mathrm{N} 4 \mathrm{~A}$ & $1.310(8)$ & $\mathrm{C} 8 \mathrm{~B}-\mathrm{H} 8 \mathrm{~F}$ & 0.9800 \\
$\mathrm{C} 7 \mathrm{~A}-\mathrm{N} 3 \mathrm{~A}$ & $1.378(8)$ & $\mathrm{N} 1 \mathrm{~B}-\mathrm{N} 2 \mathrm{~B}$ & $1.385(7)$ \\
$\mathrm{C} 7 \mathrm{~A}-\mathrm{C} 8 \mathrm{~A}$ & $1.489(8)$ & $\mathrm{N} 1 \mathrm{~B}-\mathrm{Cu}$ & $1.951(5)$ \\
$\mathrm{C} 8 \mathrm{~A}-\mathrm{H} 8 \mathrm{~A}$ & $\mathrm{~N} 3 \mathrm{~B}-\mathrm{H} 3 \mathrm{NB}$ & $0.85(3)$ \\
$\mathrm{C} 8 \mathrm{~A}-\mathrm{H} 8 \mathrm{~B}$ & 0.9800 & $\mathrm{~N} 4 \mathrm{~B}-\mathrm{N} 5 \mathrm{~B}$ & $1.391(6)$ \\
$\mathrm{C} 8 \mathrm{~A}-\mathrm{H} 8 \mathrm{C}$ & 0.9800 & $\mathrm{~N} 6 \mathrm{~B}-\mathrm{Cu}$ & $2.125(5)$ \\
N1A-N2A & 0.9800 & $\mathrm{O} 1-\mathrm{Cu}$ & $2.410(5)$ \\
N1A-Cu & $1.382(7)$ & $\mathrm{O} 1-\mathrm{H} 1 \mathrm{~W}$ & $0.83(3)$ \\
N3A-H3NA & $1.953(5)$ & $\mathrm{O} 1-\mathrm{H} 2 \mathrm{~W}$ & $0.83(3)$ \\
& $0.86(3)$ & &
\end{tabular}




$$
\begin{aligned}
& \mathrm{N} 4 \mathrm{~A}-\mathrm{N} 5 \mathrm{~A} \\
& \mathrm{~N} 6 \mathrm{~A}-\mathrm{Cu} \\
& \mathrm{C} 1 \mathrm{~B}-\mathrm{N} 6 \mathrm{~B} \\
& \mathrm{C} 1 \mathrm{~B}-\mathrm{C} 2 \mathrm{~B} \\
& \mathrm{C} 1 \mathrm{~B}-\mathrm{C} 5 \mathrm{~B} \\
& \mathrm{C} 2 \mathrm{~B}-\mathrm{N} 7 \mathrm{~B} \\
& \mathrm{C} 2 \mathrm{~B}-\mathrm{H} 2 \mathrm{~B}
\end{aligned}
$$

$\mathrm{N} 6 \mathrm{~A}-\mathrm{C} 1 \mathrm{~A}-\mathrm{C} 4 \mathrm{~A}$

$\mathrm{N} 6 \mathrm{~A}-\mathrm{C} 1 \mathrm{~A}-\mathrm{C} 5 \mathrm{~A}$

$\mathrm{C} 4 \mathrm{~A}-\mathrm{C} 1 \mathrm{~A}-\mathrm{C} 5 \mathrm{~A}$

$\mathrm{N} 6 \mathrm{~A}-\mathrm{C} 2 \mathrm{~A}-\mathrm{C} 3 \mathrm{~A}$

$\mathrm{N} 6 \mathrm{~A}-\mathrm{C} 2 \mathrm{~A}-\mathrm{H} 2 \mathrm{~A}$

$\mathrm{C} 3 \mathrm{~A}-\mathrm{C} 2 \mathrm{~A}-\mathrm{H} 2 \mathrm{~A}$

$\mathrm{N} 7 \mathrm{~A}-\mathrm{C} 3 \mathrm{~A}-\mathrm{C} 2 \mathrm{~A}$

$\mathrm{N} 7 \mathrm{~A}-\mathrm{C} 3 \mathrm{~A}-\mathrm{H} 3 \mathrm{~A}$

$\mathrm{C} 2 \mathrm{~A}-\mathrm{C} 3 \mathrm{~A}-\mathrm{H} 3 \mathrm{~A}$

$\mathrm{N} 7 \mathrm{~A}-\mathrm{C} 4 \mathrm{~A}-\mathrm{C} 1 \mathrm{~A}$

N7A-C4A-H4A

$\mathrm{C} 1 \mathrm{~A}-\mathrm{C} 4 \mathrm{~A}-\mathrm{H} 4 \mathrm{~A}$

$\mathrm{N} 1 \mathrm{~A}-\mathrm{C} 5 \mathrm{~A}-\mathrm{N} 5 \mathrm{~A}$

$\mathrm{N} 1 \mathrm{~A}-\mathrm{C} 5 \mathrm{~A}-\mathrm{C} 1 \mathrm{~A}$

$\mathrm{N} 5 \mathrm{~A}-\mathrm{C} 5 \mathrm{~A}-\mathrm{C} 1 \mathrm{~A}$

$\mathrm{N} 2 \mathrm{~A}-\mathrm{C} 6 \mathrm{~A}-\mathrm{N} 5 \mathrm{~A}$

$\mathrm{N} 2 \mathrm{~A}-\mathrm{C} 6 \mathrm{~A}-\mathrm{N} 3 \mathrm{~A}$

$\mathrm{N} 5 \mathrm{~A}-\mathrm{C} 6 \mathrm{~A}-\mathrm{N} 3 \mathrm{~A}$

$\mathrm{N} 4 \mathrm{~A}-\mathrm{C} 7 \mathrm{~A}-\mathrm{N} 3 \mathrm{~A}$

$\mathrm{N} 4 \mathrm{~A}-\mathrm{C} 7 \mathrm{~A}-\mathrm{C} 8 \mathrm{~A}$

$\mathrm{N} 3 \mathrm{~A}-\mathrm{C} 7 \mathrm{~A}-\mathrm{C} 8 \mathrm{~A}$

$\mathrm{C} 7 \mathrm{~A}-\mathrm{C} 8 \mathrm{~A}-\mathrm{H} 8 \mathrm{~A}$

$\mathrm{C} 7 \mathrm{~A}-\mathrm{C} 8 \mathrm{~A}-\mathrm{H} 8 \mathrm{~B}$

$\mathrm{H} 8 \mathrm{~A}-\mathrm{C} 8 \mathrm{~A}-\mathrm{H} 8 \mathrm{~B}$

$\mathrm{C} 7 \mathrm{~A}-\mathrm{C} 8 \mathrm{~A}-\mathrm{H} 8 \mathrm{C}$

$\mathrm{H} 8 \mathrm{~A}-\mathrm{C} 8 \mathrm{~A}-\mathrm{H} 8 \mathrm{C}$

$\mathrm{H} 8 \mathrm{~B}-\mathrm{C} 8 \mathrm{~A}-\mathrm{H} 8 \mathrm{C}$

$\mathrm{C} 5 \mathrm{~A}-\mathrm{N} 1 \mathrm{~A}-\mathrm{N} 2 \mathrm{~A}$

$\mathrm{C} 5 \mathrm{~A}-\mathrm{N} 1 \mathrm{~A}-\mathrm{Cu}$

$\mathrm{N} 2 \mathrm{~A}-\mathrm{N} 1 \mathrm{~A}-\mathrm{Cu}$

$\mathrm{C} 6 \mathrm{~A}-\mathrm{N} 2 \mathrm{~A}-\mathrm{N} 1 \mathrm{~A}$

$\mathrm{C} 6 \mathrm{~A}-\mathrm{N} 3 \mathrm{~A}-\mathrm{C} 7 \mathrm{~A}$

$\mathrm{C} 6 \mathrm{~A}-\mathrm{N} 3 \mathrm{~A}-\mathrm{H} 3 \mathrm{NA}$

$\mathrm{C} 7 \mathrm{~A}-\mathrm{N} 3 \mathrm{~A}-\mathrm{H} 3 \mathrm{NA}$

$\mathrm{C} 7 \mathrm{~A}-\mathrm{N} 4 \mathrm{~A}-\mathrm{N} 5 \mathrm{~A}$

$\mathrm{C} 6 \mathrm{~A}-\mathrm{N} 5 \mathrm{~A}-\mathrm{C} 5 \mathrm{~A}$

$\mathrm{C} 6 \mathrm{~A}-\mathrm{N} 5 \mathrm{~A}-\mathrm{N} 4 \mathrm{~A}$

$\mathrm{C} 5 \mathrm{~A}-\mathrm{N} 5 \mathrm{~A}-\mathrm{N} 4 \mathrm{~A}$

$\mathrm{C} 2 \mathrm{~A}-\mathrm{N} 6 \mathrm{~A}-\mathrm{C} 1 \mathrm{~A}$

$\mathrm{C} 2 \mathrm{~A}-\mathrm{N} 6 \mathrm{~A}-\mathrm{Cu}$
$1.380(7)$

$2.117(5)$

$1.343(7)$

$1.374(8)$

$1.444(8)$

$1.331(8)$

0.9500

$121.1(5)$

$113.4(5)$

$125.5(5)$

$120.0(6)$

120.0

120.0

$122.6(6)$

118.7

118.7

$121.6(6)$

119.2

119.2

$107.3(5)$

$120.2(5)$

$132.5(5)$

$113.6(5)$

$141.3(5)$

$105.0(5)$

$114.4(5)$

$123.9(6)$

$121.8(6)$

109.5

109.5

109.5

109.5

109.5

109.5

$111.8(5)$

$114.2(4)$

$133.9(4)$

$102.1(5)$

$105.4(5)$

$128(5)$

$126(5)$

$101.5(5)$

$105.2(5)$

$113.7(5)$

$141.1(5)$

$117.8(5)$

$130.8(4)$
$\mathrm{O} 2-\mathrm{H} 3 \mathrm{~W}$

$\mathrm{O} 2-\mathrm{H} 4 \mathrm{~W}$

$\mathrm{O} 3-\mathrm{H} 5 \mathrm{~W}$

$\mathrm{O} 3-\mathrm{H} 6 \mathrm{~W}$

O4-H7W

O4- $\mathrm{H} 8 \mathrm{~W}$

$\mathrm{Br} 1-\mathrm{Cu}$

$\mathrm{C} 3 \mathrm{~B}-\mathrm{C} 4 \mathrm{~B}-\mathrm{H} 4 \mathrm{~B}$

$\mathrm{N} 1 \mathrm{~B}-\mathrm{C} 5 \mathrm{~B}-\mathrm{N} 5 \mathrm{~B}$

$\mathrm{N} 1 \mathrm{~B}-\mathrm{C} 5 \mathrm{~B}-\mathrm{C} 1 \mathrm{~B}$

$\mathrm{N} 5 \mathrm{~B}-\mathrm{C} 5 \mathrm{~B}-\mathrm{C} 1 \mathrm{~B}$

$\mathrm{N} 2 \mathrm{~B}-\mathrm{C} 6 \mathrm{~B}-\mathrm{N} 5 \mathrm{~B}$

$\mathrm{N} 2 \mathrm{~B}-\mathrm{C} 6 \mathrm{~B}-\mathrm{N} 3 \mathrm{~B}$

$\mathrm{N} 5 \mathrm{~B}-\mathrm{C} 6 \mathrm{~B}-\mathrm{N} 3 \mathrm{~B}$

$\mathrm{N} 4 \mathrm{~B}-\mathrm{C} 7 \mathrm{~B}-\mathrm{N} 3 \mathrm{~B}$

$\mathrm{N} 4 \mathrm{~B}-\mathrm{C} 7 \mathrm{~B}-\mathrm{C} 8 \mathrm{~B}$

$\mathrm{N} 3 \mathrm{~B}-\mathrm{C} 7 \mathrm{~B}-\mathrm{C} 8 \mathrm{~B}$

$\mathrm{C} 7 \mathrm{~B}-\mathrm{C} 8 \mathrm{~B}-\mathrm{H} 8 \mathrm{D}$

$\mathrm{C} 7 \mathrm{~B}-\mathrm{C} 8 \mathrm{~B}-\mathrm{H} 8 \mathrm{E}$

$\mathrm{H} 8 \mathrm{D}-\mathrm{C} 8 \mathrm{~B}-\mathrm{H} 8 \mathrm{E}$

$\mathrm{C} 7 \mathrm{~B}-\mathrm{C} 8 \mathrm{~B}-\mathrm{H} 8 \mathrm{~F}$

$\mathrm{H} 8 \mathrm{D}-\mathrm{C} 8 \mathrm{~B}-\mathrm{H} 8 \mathrm{~F}$

$\mathrm{H} 8 \mathrm{E}-\mathrm{C} 8 \mathrm{~B}-\mathrm{H} 8 \mathrm{~F}$

$\mathrm{C} 5 \mathrm{~B}-\mathrm{N} 1 \mathrm{~B}-\mathrm{N} 2 \mathrm{~B}$

$\mathrm{C} 5 \mathrm{~B}-\mathrm{N} 1 \mathrm{~B}-\mathrm{Cu}$

$\mathrm{N} 2 \mathrm{~B}-\mathrm{N} 1 \mathrm{~B}-\mathrm{Cu}$

$\mathrm{C} 6 \mathrm{~B}-\mathrm{N} 2 \mathrm{~B}-\mathrm{N} 1 \mathrm{~B}$

$\mathrm{C} 6 \mathrm{~B}-\mathrm{N} 3 \mathrm{~B}-\mathrm{C} 7 \mathrm{~B}$

$\mathrm{C} 6 \mathrm{~B}-\mathrm{N} 3 \mathrm{~B}-\mathrm{H} 3 \mathrm{NB}$

$\mathrm{C} 7 \mathrm{~B}-\mathrm{N} 3 \mathrm{~B}-\mathrm{H} 3 \mathrm{NB}$

$\mathrm{C} 7 \mathrm{~B}-\mathrm{N} 4 \mathrm{~B}-\mathrm{N} 5 \mathrm{~B}$

$\mathrm{C} 6 \mathrm{~B}-\mathrm{N} 5 \mathrm{~B}-\mathrm{C} 5 \mathrm{~B}$

$\mathrm{C} 6 \mathrm{~B}-\mathrm{N} 5 \mathrm{~B}-\mathrm{N} 4 \mathrm{~B}$

$\mathrm{C} 5 \mathrm{~B}-\mathrm{N} 5 \mathrm{~B}-\mathrm{N} 4 \mathrm{~B}$

$\mathrm{C} 4 \mathrm{~B}-\mathrm{N} 6 \mathrm{~B}-\mathrm{C} 1 \mathrm{~B}$

$\mathrm{C} 4 \mathrm{~B}-\mathrm{N} 6 \mathrm{~B}-\mathrm{Cu}$

$\mathrm{C} 1 \mathrm{~B}-\mathrm{N} 6 \mathrm{~B}-\mathrm{Cu}$

$\mathrm{C} 3 \mathrm{~B}-\mathrm{N} 7 \mathrm{~B}-\mathrm{C} 2 \mathrm{~B}$

$\mathrm{Cu}-\mathrm{O} 1-\mathrm{H} 1 \mathrm{~W}$

$\mathrm{Cu}-\mathrm{O} 1-\mathrm{H} 2 \mathrm{~W}$

$\mathrm{H} 1 \mathrm{~W}-\mathrm{O} 1-\mathrm{H} 2 \mathrm{~W}$

$\mathrm{H} 3 \mathrm{~W}-\mathrm{O} 2-\mathrm{H} 4 \mathrm{~W}$

$\mathrm{H} 5 \mathrm{~W}-\mathrm{O} 3-\mathrm{H} 6 \mathrm{~W}$

$\mathrm{H} 7 \mathrm{~W}-\mathrm{O} 4-\mathrm{H} 8 \mathrm{~W}$

$\mathrm{N} 1 \mathrm{~B}-\mathrm{Cu}-\mathrm{N} 1 \mathrm{~A}$

$\mathrm{N} 1 \mathrm{~B}-\mathrm{Cu}-\mathrm{N} 6 \mathrm{~A}$

$\mathrm{N} 1 \mathrm{~A}-\mathrm{Cu}-\mathrm{N} 6 \mathrm{~A}$
$0.82(3)$

$0.81(3)$

0.79 (3)

0.80 (3)

$0.83(3)$

0.81 (3)

2.7466 (12)

119.9

$106.8(5)$

$120.5(6)$

$132.6(5)$

$113.1(5)$

$141.3(5)$

$105.6(5)$

$113.6(5)$

$124.2(6)$

$122.1(6)$

109.5

109.5

109.5

109.5

109.5

109.5

$111.6(5)$

114.2 (4)

134.2 (4)

102.5 (5)

106.2 (5)

119 (5)

134 (5)

$101.8(5)$

$106.0(5)$

112.7 (5)

$141.2(5)$

117.2 (5)

131.1 (4)

111.7 (4)

$116.5(5)$

$111(6)$

115 (6)

101 (4)

112 (9)

103 (8)

105 (9)

175.5 (2)

97.78 (19)

80.67 (19) 


\begin{tabular}{|c|c|}
\hline $\mathrm{C} 1 \mathrm{~A}-\mathrm{N} 6 \mathrm{~A}-\mathrm{Cu}$ & $111.4(4)$ \\
\hline $\mathrm{C} 3 \mathrm{~A}-\mathrm{N} 7 \mathrm{~A}-\mathrm{C} 4 \mathrm{~A}$ & $116.8(5)$ \\
\hline $\mathrm{N} 6 \mathrm{~B}-\mathrm{C} 1 \mathrm{~B}-\mathrm{C} 2 \mathrm{~B}$ & $122.2(5)$ \\
\hline $\mathrm{N} 6 \mathrm{~B}-\mathrm{C} 1 \mathrm{~B}-\mathrm{C} 5 \mathrm{~B}$ & $113.0(5)$ \\
\hline $\mathrm{C} 2 \mathrm{~B}-\mathrm{C} 1 \mathrm{~B}-\mathrm{C} 5 \mathrm{~B}$ & $124.8(5)$ \\
\hline $\mathrm{N} 7 \mathrm{~B}-\mathrm{C} 2 \mathrm{~B}-\mathrm{C} 1 \mathrm{~B}$ & $121.4(6)$ \\
\hline $\mathrm{N} 7 \mathrm{~B}-\mathrm{C} 2 \mathrm{~B}-\mathrm{H} 2 \mathrm{~B}$ & 119.3 \\
\hline $\mathrm{C} 1 \mathrm{~B}-\mathrm{C} 2 \mathrm{~B}-\mathrm{H} 2 \mathrm{~B}$ & 119.3 \\
\hline $\mathrm{N} 7 \mathrm{~B}-\mathrm{C} 3 \mathrm{~B}-\mathrm{C} 4 \mathrm{~B}$ & $122.5(6)$ \\
\hline $\mathrm{N} 7 \mathrm{~B}-\mathrm{C} 3 \mathrm{~B}-\mathrm{H} 3 \mathrm{~B}$ & 118.7 \\
\hline $\mathrm{C} 4 \mathrm{~B}-\mathrm{C} 3 \mathrm{~B}-\mathrm{H} 3 \mathrm{~B}$ & 118.7 \\
\hline $\mathrm{N} 6 \mathrm{~B}-\mathrm{C} 4 \mathrm{~B}-\mathrm{C} 3 \mathrm{~B}$ & $120.2(6)$ \\
\hline $\mathrm{N} 6 \mathrm{~B}-\mathrm{C} 4 \mathrm{~B}-\mathrm{H} 4 \mathrm{~B}$ & 119.9 \\
\hline $\mathrm{N} 6 \mathrm{~A}-\mathrm{C} 2 \mathrm{~A}-\mathrm{C} 3 \mathrm{~A}-\mathrm{N} 7 \mathrm{~A}$ & $1.2(9)$ \\
\hline $\mathrm{N} 6 \mathrm{~A}-\mathrm{C} 1 \mathrm{~A}-\mathrm{C} 4 \mathrm{~A}-\mathrm{N} 7 \mathrm{~A}$ & $-1.6(9)$ \\
\hline $\mathrm{C} 5 \mathrm{~A}-\mathrm{C} 1 \mathrm{~A}-\mathrm{C} 4 \mathrm{~A}-\mathrm{N} 7 \mathrm{~A}$ & $179.5(5)$ \\
\hline $\mathrm{N} 6 \mathrm{~A}-\mathrm{C} 1 \mathrm{~A}-\mathrm{C} 5 \mathrm{~A}-\mathrm{N} 1 \mathrm{~A}$ & $2.0(8)$ \\
\hline $\mathrm{C} 4 \mathrm{~A}-\mathrm{C} 1 \mathrm{~A}-\mathrm{C} 5 \mathrm{~A}-\mathrm{N} 1 \mathrm{~A}$ & $-179.0(6)$ \\
\hline $\mathrm{N} 6 \mathrm{~A}-\mathrm{C} 1 \mathrm{~A}-\mathrm{C} 5 \mathrm{~A}-\mathrm{N} 5 \mathrm{~A}$ & $-179.4(6)$ \\
\hline $\mathrm{C} 4 \mathrm{~A}-\mathrm{C} 1 \mathrm{~A}-\mathrm{C} 5 \mathrm{~A}-\mathrm{N} 5 \mathrm{~A}$ & $-0.5(10)$ \\
\hline $\mathrm{N} 5 \mathrm{~A}-\mathrm{C} 5 \mathrm{~A}-\mathrm{N} 1 \mathrm{~A}-\mathrm{N} 2 \mathrm{~A}$ & $0.1(6)$ \\
\hline $\mathrm{C} 1 \mathrm{~A}-\mathrm{C} 5 \mathrm{~A}-\mathrm{N} 1 \mathrm{~A}-\mathrm{N} 2 \mathrm{~A}$ & $179.0(5)$ \\
\hline $\mathrm{N} 5 \mathrm{~A}-\mathrm{C} 5 \mathrm{~A}-\mathrm{N} 1 \mathrm{~A}-\mathrm{Cu}$ & $177.7(3)$ \\
\hline $\mathrm{C} 1 \mathrm{~A}-\mathrm{C} 5 \mathrm{~A}-\mathrm{N} 1 \mathrm{~A}-\mathrm{Cu}$ & $-3.4(7)$ \\
\hline $\mathrm{N} 5 \mathrm{~A}-\mathrm{C} 6 \mathrm{~A}-\mathrm{N} 2 \mathrm{~A}-\mathrm{N} 1 \mathrm{~A}$ & $0.8(6)$ \\
\hline $\mathrm{N} 3 \mathrm{~A}-\mathrm{C} 6 \mathrm{~A}-\mathrm{N} 2 \mathrm{~A}-\mathrm{N} 1 \mathrm{~A}$ & $178.5(7)$ \\
\hline $\mathrm{C} 5 \mathrm{~A}-\mathrm{N} 1 \mathrm{~A}-\mathrm{N} 2 \mathrm{~A}-\mathrm{C} 6 \mathrm{~A}$ & $-0.5(6)$ \\
\hline $\mathrm{Cu}-\mathrm{N} 1 \mathrm{~A}-\mathrm{N} 2 \mathrm{~A}-\mathrm{C} 6 \mathrm{~A}$ & $-177.5(4)$ \\
\hline $\mathrm{N} 2 \mathrm{~A}-\mathrm{C} 6 \mathrm{~A}-\mathrm{N} 3 \mathrm{~A}-\mathrm{C} 7 \mathrm{~A}$ & $-179.3(8)$ \\
\hline $\mathrm{N} 5 \mathrm{~A}-\mathrm{C} 6 \mathrm{~A}-\mathrm{N} 3 \mathrm{~A}-\mathrm{C} 7 \mathrm{~A}$ & $-1.4(6)$ \\
\hline $\mathrm{N} 4 \mathrm{~A}-\mathrm{C} 7 \mathrm{~A}-\mathrm{N} 3 \mathrm{~A}-\mathrm{C} 6 \mathrm{~A}$ & $0.7(7)$ \\
\hline $\mathrm{C} 8 \mathrm{~A}-\mathrm{C} 7 \mathrm{~A}-\mathrm{N} 3 \mathrm{~A}-\mathrm{C} 6 \mathrm{~A}$ & $-179.9(6)$ \\
\hline $\mathrm{N} 3 \mathrm{~A}-\mathrm{C} 7 \mathrm{~A}-\mathrm{N} 4 \mathrm{~A}-\mathrm{N} 5 \mathrm{~A}$ & $0.3(7)$ \\
\hline $\mathrm{C} 8 \mathrm{~A}-\mathrm{C} 7 \mathrm{~A}-\mathrm{N} 4 \mathrm{~A}-\mathrm{N} 5 \mathrm{~A}$ & $-179.1(6)$ \\
\hline $\mathrm{N} 2 \mathrm{~A}-\mathrm{C} 6 \mathrm{~A}-\mathrm{N} 5 \mathrm{~A}-\mathrm{C} 5 \mathrm{~A}$ & $-0.7(7)$ \\
\hline $\mathrm{N} 3 \mathrm{~A}-\mathrm{C} 6 \mathrm{~A}-\mathrm{N} 5 \mathrm{~A}-\mathrm{C} 5 \mathrm{~A}$ & $-179.3(4)$ \\
\hline $\mathrm{N} 2 \mathrm{~A}-\mathrm{C} 6 \mathrm{~A}-\mathrm{N} 5 \mathrm{~A}-\mathrm{N} 4 \mathrm{~A}$ & $-179.6(5)$ \\
\hline $\mathrm{N} 3 \mathrm{~A}-\mathrm{C} 6 \mathrm{~A}-\mathrm{N} 5 \mathrm{~A}-\mathrm{N} 4 \mathrm{~A}$ & $1.8(6)$ \\
\hline $\mathrm{N} 1 \mathrm{~A}-\mathrm{C} 5 \mathrm{~A}-\mathrm{N} 5 \mathrm{~A}-\mathrm{C} 6 \mathrm{~A}$ & $0.3(6)$ \\
\hline $\mathrm{C} 1 \mathrm{~A}-\mathrm{C} 5 \mathrm{~A}-\mathrm{N} 5 \mathrm{~A}-\mathrm{C} 6 \mathrm{~A}$ & $-178.4(6)$ \\
\hline $\mathrm{N} 1 \mathrm{~A}-\mathrm{C} 5 \mathrm{~A}-\mathrm{N} 5 \mathrm{~A}-\mathrm{N} 4 \mathrm{~A}$ & $178.8(6)$ \\
\hline $\mathrm{C} 1 \mathrm{~A}-\mathrm{C} 5 \mathrm{~A}-\mathrm{N} 5 \mathrm{~A}-\mathrm{N} 4 \mathrm{~A}$ & $0.1(12)$ \\
\hline $\mathrm{C} 7 \mathrm{~A}-\mathrm{N} 4 \mathrm{~A}-\mathrm{N} 5 \mathrm{~A}-\mathrm{C} 6 \mathrm{~A}$ & $-1.3(6)$ \\
\hline $\mathrm{C} 7 \mathrm{~A}-\mathrm{N} 4 \mathrm{~A}-\mathrm{N} 5 \mathrm{~A}-\mathrm{C} 5 \mathrm{~A}$ & $-179.7(7)$ \\
\hline $\mathrm{C} 3 \mathrm{~A}-\mathrm{C} 2 \mathrm{~A}-\mathrm{N} 6 \mathrm{~A}-\mathrm{C} 1 \mathrm{~A}$ & $-0.8(8)$ \\
\hline $\mathrm{C} 3 \mathrm{~A}-\mathrm{C} 2 \mathrm{~A}-\mathrm{N} 6 \mathrm{~A}-\mathrm{Cu}$ & $178.8(4)$ \\
\hline $\mathrm{C} 4 \mathrm{~A}-\mathrm{C} 1 \mathrm{~A}-\mathrm{N} 6 \mathrm{~A}-\mathrm{C} 2 \mathrm{~A}$ & $1.0(8)$ \\
\hline
\end{tabular}

$$
\begin{aligned}
& \mathrm{N} 1 \mathrm{~B}-\mathrm{Cu}-\mathrm{N} 6 \mathrm{~B} \\
& \mathrm{~N} 1 \mathrm{~A}-\mathrm{Cu}-\mathrm{N} 6 \mathrm{~B} \\
& \mathrm{~N} 6 \mathrm{~A}-\mathrm{Cu}-\mathrm{N} 6 \mathrm{~B} \\
& \mathrm{~N} 1 \mathrm{~B}-\mathrm{Cu}-\mathrm{O} 1 \\
& \mathrm{~N} 1 \mathrm{~A}-\mathrm{Cu}-\mathrm{O} 1 \\
& \mathrm{~N} 6 \mathrm{~A}-\mathrm{Cu}-\mathrm{O} 1 \\
& \mathrm{~N} 6 \mathrm{~B}-\mathrm{Cu}-\mathrm{O} 1 \\
& \mathrm{~N} 1 \mathrm{~B}-\mathrm{Cu}-\mathrm{Br} 1 \\
& \mathrm{~N} 1 \mathrm{~A}-\mathrm{Cu}-\mathrm{Br} 1 \\
& \mathrm{~N} 6 \mathrm{~A}-\mathrm{Cu}-\mathrm{Br} 1 \\
& \text { N6B-Cu-Br1 } \\
& \text { O1-Cu-Br1 }
\end{aligned}
$$$$
\mathrm{N} 6 \mathrm{~B}-\mathrm{C} 1 \mathrm{~B}-\mathrm{C} 2 \mathrm{~B}-\mathrm{N} 7 \mathrm{~B}
$$$$
\mathrm{C} 5 \mathrm{~B}-\mathrm{C} 1 \mathrm{~B}-\mathrm{C} 2 \mathrm{~B}-\mathrm{N} 7 \mathrm{~B}
$$$$
\mathrm{N} 7 \mathrm{~B}-\mathrm{C} 3 \mathrm{~B}-\mathrm{C} 4 \mathrm{~B}-\mathrm{N} 6 \mathrm{~B}
$$$$
\mathrm{N} 6 \mathrm{~B}-\mathrm{C} 1 \mathrm{~B}-\mathrm{C} 5 \mathrm{~B}-\mathrm{N} 1 \mathrm{~B}
$$$$
\mathrm{C} 2 \mathrm{~B}-\mathrm{C} 1 \mathrm{~B}-\mathrm{C} 5 \mathrm{~B}-\mathrm{N} 1 \mathrm{~B}
$$$$
\mathrm{N} 6 \mathrm{~B}-\mathrm{C} 1 \mathrm{~B}-\mathrm{C} 5 \mathrm{~B}-\mathrm{N} 5 \mathrm{~B}
$$$$
\mathrm{C} 2 \mathrm{~B}-\mathrm{C} 1 \mathrm{~B}-\mathrm{C} 5 \mathrm{~B}-\mathrm{N} 5 \mathrm{~B}
$$$$
\mathrm{N} 5 \mathrm{~B}-\mathrm{C} 5 \mathrm{~B}-\mathrm{N} 1 \mathrm{~B}-\mathrm{N} 2 \mathrm{~B}
$$$$
\mathrm{C} 1 \mathrm{~B}-\mathrm{C} 5 \mathrm{~B}-\mathrm{N} 1 \mathrm{~B}-\mathrm{N} 2 \mathrm{~B}
$$$$
\mathrm{N} 5 \mathrm{~B}-\mathrm{C} 5 \mathrm{~B}-\mathrm{N} 1 \mathrm{~B}-\mathrm{Cu}
$$$$
\mathrm{C} 1 \mathrm{~B}-\mathrm{C} 5 \mathrm{~B}-\mathrm{N} 1 \mathrm{~B}-\mathrm{Cu}
$$$$
\mathrm{N} 5 \mathrm{~B}-\mathrm{C} 6 \mathrm{~B}-\mathrm{N} 2 \mathrm{~B}-\mathrm{N} 1 \mathrm{~B}
$$$$
\mathrm{N} 3 \mathrm{~B}-\mathrm{C} 6 \mathrm{~B}-\mathrm{N} 2 \mathrm{~B}-\mathrm{N} 1 \mathrm{~B}
$$$$
\mathrm{C} 5 \mathrm{~B}-\mathrm{N} 1 \mathrm{~B}-\mathrm{N} 2 \mathrm{~B}-\mathrm{C} 6 \mathrm{~B}
$$$$
\mathrm{Cu}-\mathrm{N} 1 \mathrm{~B}-\mathrm{N} 2 \mathrm{~B}-\mathrm{C} 6 \mathrm{~B}
$$$$
\mathrm{N} 2 \mathrm{~B}-\mathrm{C} 6 \mathrm{~B}-\mathrm{N} 3 \mathrm{~B}-\mathrm{C} 7 \mathrm{~B}
$$$$
\mathrm{N} 5 \mathrm{~B}-\mathrm{C} 6 \mathrm{~B}-\mathrm{N} 3 \mathrm{~B}-\mathrm{C} 7 \mathrm{~B}
$$$$
\mathrm{N} 4 \mathrm{~B}-\mathrm{C} 7 \mathrm{~B}-\mathrm{N} 3 \mathrm{~B}-\mathrm{C} 6 \mathrm{~B}
$$$$
\mathrm{C} 8 \mathrm{~B}-\mathrm{C} 7 \mathrm{~B}-\mathrm{N} 3 \mathrm{~B}-\mathrm{C} 6 \mathrm{~B}
$$$$
\mathrm{N} 3 \mathrm{~B}-\mathrm{C} 7 \mathrm{~B}-\mathrm{N} 4 \mathrm{~B}-\mathrm{N} 5 \mathrm{~B}
$$$$
\mathrm{C} 8 \mathrm{~B}-\mathrm{C} 7 \mathrm{~B}-\mathrm{N} 4 \mathrm{~B}-\mathrm{N} 5 \mathrm{~B}
$$$$
\mathrm{N} 2 \mathrm{~B}-\mathrm{C} 6 \mathrm{~B}-\mathrm{N} 5 \mathrm{~B}-\mathrm{C} 5 \mathrm{~B}
$$$$
\mathrm{N} 3 \mathrm{~B}-\mathrm{C} 6 \mathrm{~B}-\mathrm{N} 5 \mathrm{~B}-\mathrm{C} 5 \mathrm{~B}
$$$$
\mathrm{N} 2 \mathrm{~B}-\mathrm{C} 6 \mathrm{~B}-\mathrm{N} 5 \mathrm{~B}-\mathrm{N} 4 \mathrm{~B}
$$$$
\mathrm{N} 3 \mathrm{~B}-\mathrm{C} 6 \mathrm{~B}-\mathrm{N} 5 \mathrm{~B}-\mathrm{N} 4 \mathrm{~B}
$$$$
\mathrm{N} 1 \mathrm{~B}-\mathrm{C} 5 \mathrm{~B}-\mathrm{N} 5 \mathrm{~B}-\mathrm{C} 6 \mathrm{~B}
$$$$
\mathrm{C} 1 \mathrm{~B}-\mathrm{C} 5 \mathrm{~B}-\mathrm{N} 5 \mathrm{~B}-\mathrm{C} 6 \mathrm{~B}
$$$$
\mathrm{N} 1 \mathrm{~B}-\mathrm{C} 5 \mathrm{~B}-\mathrm{N} 5 \mathrm{~B}-\mathrm{N} 4 \mathrm{~B}
$$$$
\mathrm{C} 1 \mathrm{~B}-\mathrm{C} 5 \mathrm{~B}-\mathrm{N} 5 \mathrm{~B}-\mathrm{N} 4 \mathrm{~B}
$$$$
\mathrm{C} 7 \mathrm{~B}-\mathrm{N} 4 \mathrm{~B}-\mathrm{N} 5 \mathrm{~B}-\mathrm{C} 6 \mathrm{~B}
$$$$
\mathrm{C} 7 \mathrm{~B}-\mathrm{N} 4 \mathrm{~B}-\mathrm{N} 5 \mathrm{~B}-\mathrm{C} 5 \mathrm{~B}
$$$$
\mathrm{C} 3 \mathrm{~B}-\mathrm{C} 4 \mathrm{~B}-\mathrm{N} 6 \mathrm{~B}-\mathrm{C} 1 \mathrm{~B}
$$$$
\mathrm{C} 3 \mathrm{~B}-\mathrm{C} 4 \mathrm{~B}-\mathrm{N} 6 \mathrm{~B}-\mathrm{Cu}
$$$$
\mathrm{C} 2 \mathrm{~B}-\mathrm{C} 1 \mathrm{~B}-\mathrm{N} 6 \mathrm{~B}-\mathrm{C} 4 \mathrm{~B}
$$

$80.60(19)$

$100.64(19)$

$175.62(18)$

$91.16(19)$

84.78 (19)

93.64 (17)

82.35 (17)

$92.56(15)$

$91.68(15)$

$92.06(13)$

$92.08(13)$

$172.72(11)$

$-1.4(9)$

$-178.9(5)$

$-1.9(10)$

$-1.3(8)$

$176.3(6)$

$-178.6(6)$

$-1.0(10)$

$0.3(6)$

$-177.6(5)$

$-179.9(4)$

$2.2(7)$

$-0.8(6)$

$-179.3(8)$

$0.3(6)$

$-179.4(4)$

$179.2(8)$

$0.6(6)$

$0.3(7)$

$179.9(6)$

$-1.0(6)$

$179.4(6)$

1.0 (7)

$-179.9(5)$

$179.6(5)$

$-1.3(6)$

-0.8 (6)

$176.8(6)$

-178.7 (6)

-1.2 (12)

1.4 (6)

$179.3(7)$

$0.6(8)$

179.2 (4)

$1.0(8)$ 


$\begin{array}{llll}\mathrm{C} 5 \mathrm{~A}-\mathrm{C} 1 \mathrm{~A}-\mathrm{N} 6 \mathrm{~A}-\mathrm{C} 2 \mathrm{~A} & -180.0(5) & \mathrm{C} 5 \mathrm{~B}-\mathrm{C} 1 \mathrm{~B}-\mathrm{N} 6 \mathrm{~B}-\mathrm{C} 4 \mathrm{~B} & 178.7(5) \\ \mathrm{C} 4 \mathrm{~A}-\mathrm{C} 1 \mathrm{~A}-\mathrm{N} 6 \mathrm{~A}-\mathrm{Cu} & -178.7(4) & \mathrm{C} 2 \mathrm{~B}-\mathrm{C} 1 \mathrm{~B}-\mathrm{N} 6 \mathrm{~B}-\mathrm{Cu} & -177.8(5) \\ \mathrm{C} 5 \mathrm{~A}-\mathrm{C} 1 \mathrm{~A}-\mathrm{N} 6 \mathrm{~A}-\mathrm{Cu} & 0.3(6) & \mathrm{C} 5 \mathrm{~B}-\mathrm{C} 1 \mathrm{~B}-\mathrm{N} 6 \mathrm{~B}-\mathrm{Cu} & -0.1(6) \\ \mathrm{C} 2 \mathrm{~A}-\mathrm{C} 3 \mathrm{~A}-\mathrm{N} 7 \mathrm{~A}-\mathrm{C} 4 \mathrm{~A} & -1.7(8) & \mathrm{C} 4 \mathrm{~B}-\mathrm{C} 3 \mathrm{~B}-\mathrm{N} 7 \mathrm{~B}-\mathrm{C} 2 \mathrm{~B} & 1.5(9) \\ \mathrm{C} 1 \mathrm{~A}-\mathrm{C} 4 \mathrm{~A}-\mathrm{N} 7 \mathrm{~A}-\mathrm{C} 3 \mathrm{~A} & 1.9(8) & \mathrm{C} 1 \mathrm{~B}-\mathrm{C} 2 \mathrm{~B}-\mathrm{N} 7 \mathrm{~B}-\mathrm{C} 3 \mathrm{~B} & 0.2(9)\end{array}$

Hydrogen-bond geometry $\left(\AA,{ }^{\circ}\right)$

\begin{tabular}{lllll}
\hline$D-\mathrm{H} \cdots A$ & $D-\mathrm{H}$ & $\mathrm{H} \cdots A$ & $D \cdots A$ & $D-\mathrm{H} \cdots A$ \\
\hline $\mathrm{C} 2 A-\mathrm{H} 2 A \cdots \mathrm{N} 2 B$ & 0.95 & 2.38 & $3.137(8)$ & 137 \\
$\mathrm{C} 3 A-\mathrm{H} 3 A \cdots \mathrm{Br} 1^{\mathrm{i}}$ & 0.95 & 3.11 & $4.058(6)$ & 173 \\
$\mathrm{~N} 3 A-\mathrm{H} 3 N A \cdots \mathrm{O} 2$ & $0.86(3)$ & $1.82(3)$ & $2.657(7)$ & $165(7)$ \\
$\mathrm{C} 4 B-\mathrm{H} 4 B \cdots \mathrm{N} 2 A$ & 0.95 & 2.52 & $3.276(8)$ & 136 \\
$\mathrm{C} 4 B-\mathrm{H} 4 B \cdots \mathrm{Br} 1^{\mathrm{ii}}$ & 0.95 & 3.09 & $3.848(6)$ & 138 \\
$\mathrm{~N} 3 B-\mathrm{H} 3 N B \cdots \mathrm{Br} 2$ & $0.85(3)$ & $2.34(3)$ & $3.190(5)$ & $173(7)$ \\
$\mathrm{O} 1-\mathrm{H} 1 W^{\cdots} \cdots \mathrm{Br} 1^{\text {iii }}$ & $0.83(3)$ & $2.42(3)$ & $3.229(5)$ & $166(6)$ \\
$\mathrm{O} 1-\mathrm{H} 2 W \cdots \mathrm{Br} 2^{\mathrm{ii}}$ & $0.83(3)$ & $2.49(3)$ & $3.301(5)$ & $168(8)$ \\
$\mathrm{O} 2-\mathrm{H} 3 W \cdots \mathrm{Br} 1^{\text {ii }}$ & $0.82(3)$ & $2.43(3)$ & $3.237(5)$ & $173(9)$ \\
$\mathrm{O} 2-\mathrm{H} 4 W \cdots \mathrm{O} 3^{\text {iv }}$ & $0.81(3)$ & $1.89(4)$ & $2.691(7)$ & $167(10)$ \\
$\mathrm{O} 3-\mathrm{H} 5 W \cdots \mathrm{O} 4^{\mathrm{v}}$ & $0.79(3)$ & $2.08(6)$ & $2.746(7)$ & $141(8)$ \\
$\mathrm{O} 3-\mathrm{H} 6 W \cdots \mathrm{N} 7 A$ & $0.80(3)$ & $2.17(4)$ & $2.931(7)$ & $159(9)$ \\
$\mathrm{O} 4-\mathrm{H} 7 W \cdots \mathrm{N} 7 B$ & $0.83(3)$ & $2.12(3)$ & $2.939(7)$ & $171(9)$ \\
$\mathrm{O} 4-\mathrm{H} 8 W \cdots \mathrm{Br} 2^{\text {iv }}$ & $0.81(3)$ & $2.56(6)$ & $3.272(6)$ & $147(8)$ \\
& & & &
\end{tabular}

Symmetry codes: (i) $x, y-1, z$; (ii) $x+1 / 2, y+1 / 2, z$; (iii) $x+1 / 2, y-1 / 2, z$; (iv) $x+1 / 2, y+3 / 2, z$; (v) $x,-y+1, z+1 / 2$.

Dibromido[4-methyl-7-(pyrazin-2-yl)-2H-[1,2,4] triazolo[3,2-c][1,2,4] triazole][4-methyl-7-(pyrazin-2-

yl)-3H-[1,2,4]triazolo[3,2-c][1,2,4]triazole]zinc(II) monohydrate (2)

Crystal data

$\left[\mathrm{ZnBr}_{2}\left(\mathrm{C}_{8} \mathrm{H}_{7} \mathrm{~N}_{7}\right)_{2}\right] \cdot \mathrm{H}_{2} \mathrm{O}$

$M_{r}=645.62$

Triclinic, $P \overline{1}$

$a=8.396(2) \AA$

$b=12.305(3) \AA$

$c=12.724(3) \AA$

$\alpha=112.53(2)^{\circ}$

$\beta=107.78(3)^{\circ}$

$\gamma=92.16(2)^{\circ}$

$V=1138.1(5) \AA^{3}$

\section{Data collection}

Bruker-Nonius KappaCCD diffractometer

Radiation source: normal-focus sealed tube Graphite monochromator

Detector resolution: 9 pixels $\mathrm{mm}^{-1}$

CCD rotation images, thick slices scans

Absorption correction: multi-scan

(SADABS; Bruker, 2001)

$T_{\min }=0.270, T_{\max }=0.433$
$Z=2$

$F(000)=636$

$D_{\mathrm{x}}=1.884 \mathrm{Mg} \mathrm{m}^{-3}$

Mo $K \alpha$ radiation, $\lambda=0.71073 \AA$

Cell parameters from 467 reflections

$\theta=2.5-29.4^{\circ}$

$\mu=4.63 \mathrm{~mm}^{-1}$

$T=293 \mathrm{~K}$

Prismatic, brown

$0.40 \times 0.30 \times 0.20 \mathrm{~mm}$

12306 measured reflections

5090 independent reflections

3636 reflections with $I>2 \sigma(I)$

$R_{\text {int }}=0.042$

$\theta_{\max }=27.5^{\circ}, \theta_{\min }=3.0^{\circ}$

$h=-10 \rightarrow 9$

$k=-15 \rightarrow 15$

$l=-16 \rightarrow 16$ 


\section{Refinement}

Refinement on $F^{2}$

Least-squares matrix: full

$R\left[F^{2}>2 \sigma\left(F^{2}\right)\right]=0.047$

$w R\left(F^{2}\right)=0.136$

$S=1.09$

5090 reflections

315 parameters

2 restraints

Primary atom site location: structure-invariant direct methods
Secondary atom site location: difference Fourier map

Hydrogen site location: mixed

$\mathrm{H}$ atoms treated by a mixture of independent and constrained refinement

$w=1 /\left[\sigma^{2}\left(F_{\mathrm{o}}^{2}\right)+(0.0621 P)^{2}+1.6216 P\right]$ where $P=\left(F_{\mathrm{o}}^{2}+2 F_{\mathrm{c}}^{2}\right) / 3$

$(\Delta / \sigma)_{\max }<0.001$

$\Delta \rho_{\max }=0.70 \mathrm{e} \AA^{-3}$

$\Delta \rho_{\min }=-0.74$ e $\AA^{-3}$

Special details

Geometry. All esds (except the esd in the dihedral angle between two 1.s. planes) are estimated using the full covariance matrix. The cell esds are taken into account individually in the estimation of esds in distances, angles and torsion angles; correlations between esds in cell parameters are only used when they are defined by crystal symmetry. An approximate (isotropic) treatment of cell esds is used for estimating esds involving 1.s. planes.

Refinement. Data were collected on a Bruker-Nonius KappaCCD diffractometer equipped with Oxford Cryostream 700 apparatus. The structures were solved by direct methods and refined by the full matrix least squares method with anisotropic displacement parameters for non-H atoms.

Fractional atomic coordinates and isotropic or equivalent isotropic displacement parameters $\left(\AA^{2}\right)$

\begin{tabular}{|c|c|c|c|c|}
\hline & $x$ & $y$ & $z$ & $U_{\text {iso }} * / U_{\text {eq }}$ \\
\hline C1A & $0.7622(6)$ & $-0.1592(4)$ & $0.2765(5)$ & $0.0327(10)$ \\
\hline $\mathrm{C} 2 \mathrm{~A}$ & $0.8753(7)$ & $-0.1982(5)$ & $0.3538(5)$ & $0.0419(12)$ \\
\hline $\mathrm{H} 2 \mathrm{~A}$ & 0.910796 & -0.152912 & 0.437155 & $0.050 *$ \\
\hline $\mathrm{C} 3 \mathrm{~A}$ & $0.8825(7)$ & $-0.3578(5)$ & $0.1909(6)$ & 0.0473 (13) \\
\hline $\mathrm{H} 3 \mathrm{~A}$ & 0.919272 & -0.429248 & 0.157203 & $0.057^{*}$ \\
\hline $\mathrm{C} 4 \mathrm{~A}$ & $0.7757(8)$ & $-0.3151(5)$ & $0.1150(6)$ & 0.0499 (14) \\
\hline $\mathrm{H} 4 \mathrm{~A}$ & 0.747553 & -0.357230 & 0.031646 & $0.060 *$ \\
\hline C5A & $0.6867(6)$ & $-0.0542(4)$ & $0.3258(5)$ & $0.0328(10)$ \\
\hline C6A & $0.5263(6)$ & $0.0829(4)$ & $0.3413(4)$ & $0.0323(10)$ \\
\hline C7A & $0.3906(6)$ & $0.0624(5)$ & $0.1628(5)$ & $0.0394(11)$ \\
\hline C8A & $0.2693(8)$ & $0.0807(7)$ & $0.0606(5)$ & $0.0581(17)$ \\
\hline H8A & 0.273891 & 0.023930 & -0.014849 & $0.087^{*}$ \\
\hline H8B & 0.299509 & 0.160286 & 0.068690 & $0.087^{*}$ \\
\hline $\mathrm{H} 8 \mathrm{C}$ & 0.156311 & 0.069745 & 0.061995 & $0.087^{*}$ \\
\hline N1A & $0.7145(5)$ & $0.0033(4)$ & $0.4421(4)$ & $0.0394(10)$ \\
\hline $\mathrm{N} 2 \mathrm{~A}$ & $0.6147(6)$ & 0.0897 (4) & $0.4513(4)$ & $0.0391(10)$ \\
\hline H2NA & $0.607(7)$ & $0.138(4)$ & $0.517(3)$ & $0.047^{*}$ \\
\hline N3A & $0.4115(5)$ & $0.1312(4)$ & $0.2834(4)$ & $0.0352(9)$ \\
\hline N4A & $0.4836(5)$ & -0.0220 & $0.1438(4)$ & $0.0394(10)$ \\
\hline N5A & $0.5711(5)$ & $-0.0065(4)$ & $0.2614(4)$ & $0.0334(9)$ \\
\hline N6A & $0.7115(6)$ & $-0.2166(4)$ & $0.1556(4)$ & $0.0445(11)$ \\
\hline N7A & $0.9342(6)$ & -0.2990 & $0.3114(5)$ & $0.0472(11)$ \\
\hline $\mathrm{C} 1 \mathrm{~B}$ & $0.6464(6)$ & $0.3015(4)$ & $0.7817(4)$ & $0.0335(10)$ \\
\hline $\mathrm{C} 2 \mathrm{~B}$ & $0.7013(8)$ & $0.3681(5)$ & $0.9056(5)$ & $0.0525(15)$ \\
\hline $\mathrm{H} 2 \mathrm{~B}$ & 0.653069 & 0.434809 & 0.935948 & $0.063^{*}$ \\
\hline $\mathrm{C} 3 \mathrm{~B}$ & $0.8740(8)$ & $0.2402(6)$ & $0.9334(6)$ & $0.0560(16)$ \\
\hline
\end{tabular}




$\begin{array}{lllll}\text { H3B } & 0.954592 & 0.215786 & 0.984276 & 0.067^{*} \\ \text { C4B } & 0.8170(7) & 0.1699(5) & 0.8100(6) & 0.0476(14) \\ \text { H4B } & 0.860005 & 0.100278 & 0.780550 & 0.057^{*} \\ \text { C5B } & 0.5245(6) & 0.3357(4) & 0.6953(4) & 0.0333(10) \\ \text { C6B } & 0.3573(6) & 0.4326(4) & 0.6126(5) & 0.0364(11) \\ \text { C7B } & 0.3548(6) & 0.5944(4) & 0.7617(5) & 0.0352(10) \\ \text { C8B } & 0.3150(7) & 0.7132(5) & 0.8251(6) & 0.0474(13) \\ \text { H8D } & 0.201770 & 0.703714 & 0.826530 & 0.071^{*} \\ \text { H8E } & 0.323254 & 0.762347 & 0.783202 & 0.071^{*} \\ \text { H8F } & 0.394277 & 0.750544 & 0.906771 & 0.071^{*} \\ \text { N1B } & 0.4712(6) & 0.2739(4) & 0.5773(4) & 0.0408(10) \\ \text { N2B } & 0.3645(5) & 0.3337(4) & 0.5216(4) & 0.0412(10) \\ \text { N3B } & 0.2928(5) & 0.5339(4) & 0.6371(4) & 0.0376(10) \\ \text { H3NB } & 0.245(6) & 0.563(5) & 0.588(4) & 0.045^{*} \\ \text { N4B } & 0.4512(5) & 0.5378(4) & 0.8169(4) & 0.0351(9) \\ \text { N5B } & 0.4513(5) & 0.4356(3) & 0.7202(3) & 0.0310(8) \\ \text { N6B } & 0.7007(5) & 0.2004(4) & 0.7322(4) & 0.0388(10) \\ \text { N7B } & 0.8197(7) & 0.3407(5) & 0.9827(5) & 0.0585(14) \\ \text { Zn } & 0.25393(7) & 0.24715(5) & 0.33982(5) & 0.03379(16) \\ \text { Br1 } & 0.00038(7) & 0.11388(5) & 0.29176(6) & 0.04583(17) \\ \text { Br2 } & 0.20342(8) & 0.39584(6) & 0.26552(7) & 0.0577(2) \\ \text { O1W } & 0.1184(9) & 0.6539(6) & 0.5184(5) & 0.103(2) \\ \text { H1W } & 0.061280 & 0.710175 & 0.572921 & 0.124^{*} \\ \text { H2W } & 0.050768 & 0.636549 & 0.434186 & 0.124^{*} \\ & & & & \end{array}$

Atomic displacement parameters $\left(\AA^{2}\right)$

\begin{tabular}{lllllll}
\hline & $U^{11}$ & $U^{22}$ & $U^{33}$ & $U^{12}$ & $U^{13}$ & $U^{23}$ \\
\hline C1A & $0.034(2)$ & $0.031(2)$ & $0.036(3)$ & $0.0062(18)$ & $0.013(2)$ & $0.015(2)$ \\
C2A & $0.044(3)$ & $0.046(3)$ & $0.038(3)$ & $0.013(2)$ & $0.015(2)$ & $0.019(3)$ \\
C3A & $0.052(3)$ & $0.036(3)$ & $0.053(4)$ & $0.015(2)$ & $0.022(3)$ & $0.014(3)$ \\
C4A & $0.058(3)$ & $0.042(3)$ & $0.040(3)$ & $0.009(3)$ & $0.014(3)$ & $0.009(3)$ \\
C5A & $0.033(2)$ & $0.033(2)$ & $0.034(3)$ & $0.0084(19)$ & $0.012(2)$ & $0.016(2)$ \\
C6A & $0.030(2)$ & $0.035(2)$ & $0.029(3)$ & $0.0062(18)$ & $0.0089(19)$ & $0.010(2)$ \\
C7A & $0.034(2)$ & $0.051(3)$ & $0.033(3)$ & $0.010(2)$ & $0.011(2)$ & $0.018(2)$ \\
C8A & $0.056(4)$ & $0.081(5)$ & $0.034(3)$ & $0.032(3)$ & $0.012(3)$ & $0.022(3)$ \\
N1A & $0.046(2)$ & $0.039(2)$ & $0.037(2)$ & $0.0150(18)$ & $0.0148(19)$ & $0.018(2)$ \\
N2A & $0.046(2)$ & $0.041(2)$ & $0.028(2)$ & $0.0166(19)$ & $0.0121(19)$ & $0.0122(19)$ \\
N3A & $0.033(2)$ & $0.044(2)$ & $0.030(2)$ & $0.0121(17)$ & $0.0098(17)$ & $0.0169(19)$ \\
N4A & $0.041(2)$ & $0.046(2)$ & $0.030(2)$ & $0.0111(18)$ & $0.0093(18)$ & $0.015(2)$ \\
N5A & $0.033(2)$ & $0.035(2)$ & $0.031(2)$ & $0.0075(16)$ & $0.0090(17)$ & $0.0143(18)$ \\
N6A & $0.050(3)$ & $0.040(2)$ & $0.038(3)$ & $0.009(2)$ & $0.010(2)$ & $0.015(2)$ \\
N7A & $0.054(3)$ & $0.044(3)$ & $0.049(3)$ & $0.018(2)$ & $0.019(2)$ & $0.022(2)$ \\
C1B & $0.037(2)$ & $0.031(2)$ & $0.031(3)$ & $0.0064(19)$ & $0.007(2)$ & $0.013(2)$ \\
C2B & $0.064(4)$ & $0.045(3)$ & $0.034(3)$ & $0.020(3)$ & $0.001(3)$ & $0.013(3)$ \\
C3B & $0.057(4)$ & $0.064(4)$ & $0.053(4)$ & $0.018(3)$ & $0.008(3)$ & $0.038(3)$ \\
C4B & $0.046(3)$ & $0.041(3)$ & $0.060(4)$ & $0.017(2)$ & $0.016(3)$ & $0.027(3)$ \\
C5B & $0.036(2)$ & $0.029(2)$ & $0.030(3)$ & $0.0048(18)$ & $0.009(2)$ & $0.009(2)$
\end{tabular}


supporting information

$\begin{array}{lllllll} & & & & & \\ \text { C6B } & 0.036(2) & 0.036(3) & 0.033(3) & 0.0078(19) & 0.006(2) & 0.014(2) \\ \text { C7B } & 0.032(2) & 0.038(3) & 0.038(3) & 0.0065(19) & 0.015(2) & 0.016(2) \\ \text { C8B } & 0.055(3) & 0.039(3) & 0.052(4) & 0.022(2) & 0.024(3) & 0.018(3) \\ \text { N1B } & 0.047(2) & 0.037(2) & 0.032(2) & 0.0122(18) & 0.0070(19) & 0.0116(19) \\ \text { N2B } & 0.043(2) & 0.039(2) & 0.034(2) & 0.0134(18) & 0.0041(19) & 0.013(2) \\ \text { N3B } & 0.037(2) & 0.040(2) & 0.036(2) & 0.0131(18) & 0.0079(18) & 0.019(2) \\ \text { N4B } & 0.040(2) & 0.032(2) & 0.029(2) & 0.0082(17) & 0.0104(17) & 0.0097(18) \\ \text { N5B } & 0.036(2) & 0.0290(19) & 0.023(2) & 0.0067(16) & 0.0060(16) & 0.0093(16) \\ \text { N6B } & 0.038(2) & 0.034(2) & 0.042(3) & 0.0103(17) & 0.0131(19) & 0.013(2) \\ \text { N7B } & 0.062(3) & 0.068(3) & 0.040(3) & 0.025(3) & 0.006(2) & 0.023(3) \\ \text { Zn } & 0.0331(3) & 0.0369(3) & 0.0277(3) & 0.0079(2) & 0.0052(2) & 0.0137(2) \\ \text { Br1 } & 0.0391(3) & 0.0392(3) & 0.0574(4) & 0.0062(2) & 0.0161(2) & 0.0187(3) \\ \text { Br2 } & 0.0535(4) & 0.0593(4) & 0.0712(5) & 0.0088(3) & 0.0129(3) & 0.0451(4) \\ \text { O1W } & 0.152(6) & 0.104(5) & 0.063(4) & 0.078(4) & 0.028(4) & 0.046(3) \\ & & & & & \end{array}$

Geometric parameters $\left(\AA,{ }^{o}\right)$

\begin{tabular}{|c|c|c|c|}
\hline $\mathrm{C} 1 \mathrm{~A}-\mathrm{N} 6 \mathrm{~A}$ & $1.338(7)$ & $\mathrm{C} 1 \mathrm{~B}-\mathrm{C} 5 \mathrm{~B}$ & $1.460(7)$ \\
\hline $\mathrm{C} 1 \mathrm{~A}-\mathrm{C} 2 \mathrm{~A}$ & $1.388(7)$ & $\mathrm{C} 2 \mathrm{~B}-\mathrm{N} 7 \mathrm{~B}$ & $1.322(7)$ \\
\hline $\mathrm{C} 1 \mathrm{~A}-\mathrm{C} 5 \mathrm{~A}$ & $1.466(7)$ & $\mathrm{C} 2 \mathrm{~B}-\mathrm{H} 2 \mathrm{~B}$ & 0.9300 \\
\hline $\mathrm{C} 2 \mathrm{~A}-\mathrm{N} 7 \mathrm{~A}$ & $1.330(7)$ & $\mathrm{C} 3 \mathrm{~B}-\mathrm{N} 7 \mathrm{~B}$ & $1.324(8)$ \\
\hline $\mathrm{C} 2 \mathrm{~A}-\mathrm{H} 2 \mathrm{~A}$ & 0.9300 & $\mathrm{C} 3 \mathrm{~B}-\mathrm{C} 4 \mathrm{~B}$ & $1.382(9)$ \\
\hline $\mathrm{C} 3 \mathrm{~A}-\mathrm{N} 7 \mathrm{~A}$ & $1.335(8)$ & $\mathrm{C} 3 \mathrm{~B}-\mathrm{H} 3 \mathrm{~B}$ & 0.9300 \\
\hline $\mathrm{C} 3 \mathrm{~A}-\mathrm{C} 4 \mathrm{~A}$ & $1.381(8)$ & $\mathrm{C} 4 \mathrm{~B}-\mathrm{N} 6 \mathrm{~B}$ & $1.340(7)$ \\
\hline $\mathrm{C} 3 \mathrm{~A}-\mathrm{H} 3 \mathrm{~A}$ & 0.9300 & $\mathrm{C} 4 \mathrm{~B}-\mathrm{H} 4 \mathrm{~B}$ & 0.9300 \\
\hline $\mathrm{C} 4 \mathrm{~A}-\mathrm{N} 6 \mathrm{~A}$ & $1.329(7)$ & $\mathrm{C} 5 \mathrm{~B}-\mathrm{N} 1 \mathrm{~B}$ & $1.313(7)$ \\
\hline $\mathrm{C} 4 \mathrm{~A}-\mathrm{H} 4 \mathrm{~A}$ & 0.9300 & $\mathrm{C} 5 \mathrm{~B}-\mathrm{N} 5 \mathrm{~B}$ & $1.366(6)$ \\
\hline $\mathrm{C} 5 \mathrm{~A}-\mathrm{N} 1 \mathrm{~A}$ & $1.310(7)$ & $\mathrm{C} 6 \mathrm{~B}-\mathrm{N} 2 \mathrm{~B}$ & $1.339(7)$ \\
\hline $\mathrm{C} 5 \mathrm{~A}-\mathrm{N} 5 \mathrm{~A}$ & $1.372(6)$ & $\mathrm{C} 6 \mathrm{~B}-\mathrm{N} 5 \mathrm{~B}$ & $1.341(6)$ \\
\hline $\mathrm{C} 6 \mathrm{~A}-\mathrm{N} 3 \mathrm{~A}$ & $1.330(6)$ & $\mathrm{C} 6 \mathrm{~B}-\mathrm{N} 3 \mathrm{~B}$ & $1.342(6)$ \\
\hline $\mathrm{C} 6 \mathrm{~A}-\mathrm{N} 2 \mathrm{~A}$ & $1.337(6)$ & $\mathrm{C} 7 \mathrm{~B}-\mathrm{N} 4 \mathrm{~B}$ & $1.305(6)$ \\
\hline $\mathrm{C} 6 \mathrm{~A}-\mathrm{N} 5 \mathrm{~A}$ & $1.339(6)$ & $\mathrm{C} 7 \mathrm{~B}-\mathrm{N} 3 \mathrm{~B}$ & $1.379(7)$ \\
\hline $\mathrm{C} 7 \mathrm{~A}-\mathrm{N} 4 \mathrm{~A}$ & $1.315(7)$ & $\mathrm{C} 7 \mathrm{~B}-\mathrm{C} 8 \mathrm{~B}$ & $1.482(7)$ \\
\hline $\mathrm{C} 7 \mathrm{~A}-\mathrm{N} 3 \mathrm{~A}$ & $1.390(7)$ & $\mathrm{C} 8 \mathrm{~B}-\mathrm{H} 8 \mathrm{D}$ & 0.9600 \\
\hline $\mathrm{C} 7 \mathrm{~A}-\mathrm{C} 8 \mathrm{~A}$ & $1.487(7)$ & $\mathrm{C} 8 \mathrm{~B}-\mathrm{H} 8 \mathrm{E}$ & 0.9600 \\
\hline $\mathrm{C} 8 \mathrm{~A}-\mathrm{H} 8 \mathrm{~A}$ & 0.9600 & $\mathrm{C} 8 \mathrm{~B}-\mathrm{H} 8 \mathrm{~F}$ & 0.9600 \\
\hline $\mathrm{C} 8 \mathrm{~A}-\mathrm{H} 8 \mathrm{~B}$ & 0.9600 & $\mathrm{~N} 1 \mathrm{~B}-\mathrm{N} 2 \mathrm{~B}$ & $1.381(6)$ \\
\hline $\mathrm{C} 8 \mathrm{~A}-\mathrm{H} 8 \mathrm{C}$ & 0.9600 & $\mathrm{~N} 2 \mathrm{~B}-\mathrm{Zn}$ & $2.018(4)$ \\
\hline $\mathrm{N} 1 \mathrm{~A}-\mathrm{N} 2 \mathrm{~A}$ & $1.371(6)$ & $\mathrm{N} 3 \mathrm{~B}-\mathrm{H} 3 \mathrm{NB}$ & $0.85(2)$ \\
\hline $\mathrm{N} 2 \mathrm{~A}-\mathrm{H} 2 \mathrm{NA}$ & $0.84(2)$ & $\mathrm{N} 4 \mathrm{~B}-\mathrm{N} 5 \mathrm{~B}$ & $1.382(6)$ \\
\hline $\mathrm{N} 3 \mathrm{~A}-\mathrm{Zn}$ & $2.059(4)$ & $\mathrm{Zn}-\mathrm{Br} 2$ & $2.3581(9)$ \\
\hline $\mathrm{N} 4 \mathrm{~A}-\mathrm{N} 5 \mathrm{~A}$ & $1.384(6)$ & $\mathrm{Zn}-\mathrm{Br} 1$ & $2.4017(11)$ \\
\hline $\mathrm{C} 1 \mathrm{~B}-\mathrm{N} 6 \mathrm{~B}$ & $1.330(6)$ & $\mathrm{O} 1 \mathrm{~W}-\mathrm{H} 1 \mathrm{~W}$ & 1.0251 \\
\hline $\mathrm{C} 1 \mathrm{~B}-\mathrm{C} 2 \mathrm{~B}$ & $1.381(8)$ & $\mathrm{O} 1 \mathrm{~W}-\mathrm{H} 2 \mathrm{~W}$ & 0.9795 \\
\hline $\mathrm{N} 6 \mathrm{~A}-\mathrm{C} 1 \mathrm{~A}-\mathrm{C} 2 \mathrm{~A}$ & $122.7(5)$ & $\mathrm{N} 7 \mathrm{~B}-\mathrm{C} 2 \mathrm{~B}-\mathrm{H} 2 \mathrm{~B}$ & 118.7 \\
\hline $\mathrm{N} 6 \mathrm{~A}-\mathrm{C} 1 \mathrm{~A}-\mathrm{C} 5 \mathrm{~A}$ & $117.0(4)$ & $\mathrm{C} 1 \mathrm{~B}-\mathrm{C} 2 \mathrm{~B}-\mathrm{H} 2 \mathrm{~B}$ & 118.7 \\
\hline $\mathrm{C} 2 \mathrm{~A}-\mathrm{C} 1 \mathrm{~A}-\mathrm{C} 5 \mathrm{~A}$ & $120.3(5)$ & $\mathrm{N} 7 \mathrm{~B}-\mathrm{C} 3 \mathrm{~B}-\mathrm{C} 4 \mathrm{~B}$ & $123.3(5)$ \\
\hline
\end{tabular}




\begin{tabular}{|c|c|}
\hline $\mathrm{N} 7 \mathrm{~A}-\mathrm{C} 2 \mathrm{~A}-\mathrm{C} 1 \mathrm{~A}$ & $121.4(5)$ \\
\hline $\mathrm{N} 7 \mathrm{~A}-\mathrm{C} 2 \mathrm{~A}-\mathrm{H} 2 \mathrm{~A}$ & 119.3 \\
\hline $\mathrm{C} 1 \mathrm{~A}-\mathrm{C} 2 \mathrm{~A}-\mathrm{H} 2 \mathrm{~A}$ & 119.3 \\
\hline $\mathrm{N} 7 \mathrm{~A}-\mathrm{C} 3 \mathrm{~A}-\mathrm{C} 4 \mathrm{~A}$ & $121.7(5)$ \\
\hline $\mathrm{N} 7 \mathrm{~A}-\mathrm{C} 3 \mathrm{~A}-\mathrm{H} 3 \mathrm{~A}$ & 119.2 \\
\hline $\mathrm{C} 4 \mathrm{~A}-\mathrm{C} 3 \mathrm{~A}-\mathrm{H} 3 \mathrm{~A}$ & 119.2 \\
\hline $\mathrm{N} 6 \mathrm{~A}-\mathrm{C} 4 \mathrm{~A}-\mathrm{C} 3 \mathrm{~A}$ & $123.0(6)$ \\
\hline $\mathrm{N} 6 \mathrm{~A}-\mathrm{C} 4 \mathrm{~A}-\mathrm{H} 4 \mathrm{~A}$ & 118.5 \\
\hline $\mathrm{C} 3 \mathrm{~A}-\mathrm{C} 4 \mathrm{~A}-\mathrm{H} 4 \mathrm{~A}$ & 118.5 \\
\hline $\mathrm{N} 1 \mathrm{~A}-\mathrm{C} 5 \mathrm{~A}-\mathrm{N} 5 \mathrm{~A}$ & $110.0(4)$ \\
\hline $\mathrm{N} 1 \mathrm{~A}-\mathrm{C} 5 \mathrm{~A}-\mathrm{C} 1 \mathrm{~A}$ & $122.7(4)$ \\
\hline $\mathrm{N} 5 \mathrm{~A}-\mathrm{C} 5 \mathrm{~A}-\mathrm{C} 1 \mathrm{~A}$ & $127.1(5)$ \\
\hline $\mathrm{N} 3 \mathrm{~A}-\mathrm{C} 6 \mathrm{~A}-\mathrm{N} 2 \mathrm{~A}$ & $143.3(5)$ \\
\hline $\mathrm{N} 3 \mathrm{~A}-\mathrm{C} 6 \mathrm{~A}-\mathrm{N} 5 \mathrm{~A}$ & $110.0(4)$ \\
\hline $\mathrm{N} 2 \mathrm{~A}-\mathrm{C} 6 \mathrm{~A}-\mathrm{N} 5 \mathrm{~A}$ & $106.7(4)$ \\
\hline $\mathrm{N} 4 \mathrm{~A}-\mathrm{C} 7 \mathrm{~A}-\mathrm{N} 3 \mathrm{~A}$ & $115.8(4)$ \\
\hline $\mathrm{N} 4 \mathrm{~A}-\mathrm{C} 7 \mathrm{~A}-\mathrm{C} 8 \mathrm{~A}$ & $121.6(5)$ \\
\hline $\mathrm{N} 3 \mathrm{~A}-\mathrm{C} 7 \mathrm{~A}-\mathrm{C} 8 \mathrm{~A}$ & $122.7(5)$ \\
\hline $\mathrm{C} 7 \mathrm{~A}-\mathrm{C} 8 \mathrm{~A}-\mathrm{H} 8 \mathrm{~A}$ & 109.5 \\
\hline $\mathrm{C} 7 \mathrm{~A}-\mathrm{C} 8 \mathrm{~A}-\mathrm{H} 8 \mathrm{~B}$ & 109.5 \\
\hline $\mathrm{H} 8 \mathrm{~A}-\mathrm{C} 8 \mathrm{~A}-\mathrm{H} 8 \mathrm{~B}$ & 109.5 \\
\hline $\mathrm{C} 7 \mathrm{~A}-\mathrm{C} 8 \mathrm{~A}-\mathrm{H} 8 \mathrm{C}$ & 109.5 \\
\hline $\mathrm{H} 8 \mathrm{~A}-\mathrm{C} 8 \mathrm{~A}-\mathrm{H} 8 \mathrm{C}$ & 109.5 \\
\hline $\mathrm{H} 8 \mathrm{~B}-\mathrm{C} 8 \mathrm{~A}-\mathrm{H} 8 \mathrm{C}$ & 109.5 \\
\hline $\mathrm{C} 5 \mathrm{~A}-\mathrm{N} 1 \mathrm{~A}-\mathrm{N} 2 \mathrm{~A}$ & $105.5(4)$ \\
\hline $\mathrm{C} 6 \mathrm{~A}-\mathrm{N} 2 \mathrm{~A}-\mathrm{N} 1 \mathrm{~A}$ & $110.4(4)$ \\
\hline $\mathrm{C} 6 \mathrm{~A}-\mathrm{N} 2 \mathrm{~A}-\mathrm{H} 2 \mathrm{NA}$ & $125(4)$ \\
\hline $\mathrm{N} 1 \mathrm{~A}-\mathrm{N} 2 \mathrm{~A}-\mathrm{H} 2 \mathrm{NA}$ & $124(4)$ \\
\hline $\mathrm{C} 6 \mathrm{~A}-\mathrm{N} 3 \mathrm{~A}-\mathrm{C} 7 \mathrm{~A}$ & $102.1(4)$ \\
\hline $\mathrm{C} 6 \mathrm{~A}-\mathrm{N} 3 \mathrm{~A}-\mathrm{Zn}$ & $131.9(4)$ \\
\hline $\mathrm{C} 7 \mathrm{~A}-\mathrm{N} 3 \mathrm{~A}-\mathrm{Zn}$ & $124.5(3)$ \\
\hline $\mathrm{C} 7 \mathrm{~A}-\mathrm{N} 4 \mathrm{~A}-\mathrm{N} 5 \mathrm{~A}$ & $101.0(4)$ \\
\hline $\mathrm{C} 6 \mathrm{~A}-\mathrm{N} 5 \mathrm{~A}-\mathrm{C} 5 \mathrm{~A}$ & $107.4(4)$ \\
\hline $\mathrm{C} 6 \mathrm{~A}-\mathrm{N} 5 \mathrm{~A}-\mathrm{N} 4 \mathrm{~A}$ & $111.0(4)$ \\
\hline $\mathrm{C} 5 \mathrm{~A}-\mathrm{N} 5 \mathrm{~A}-\mathrm{N} 4 \mathrm{~A}$ & $141.5(4)$ \\
\hline $\mathrm{C} 4 \mathrm{~A}-\mathrm{N} 6 \mathrm{~A}-\mathrm{C} 1 \mathrm{~A}$ & $114.9(5)$ \\
\hline $\mathrm{C} 2 \mathrm{~A}-\mathrm{N} 7 \mathrm{~A}-\mathrm{C} 3 \mathrm{~A}$ & $116.3(5)$ \\
\hline $\mathrm{N} 6 \mathrm{~B}-\mathrm{C} 1 \mathrm{~B}-\mathrm{C} 2 \mathrm{~B}$ & $122.1(5)$ \\
\hline $\mathrm{N} 6 \mathrm{~B}-\mathrm{C} 1 \mathrm{~B}-\mathrm{C} 5 \mathrm{~B}$ & $115.0(5)$ \\
\hline $\mathrm{C} 2 \mathrm{~B}-\mathrm{C} 1 \mathrm{~B}-\mathrm{C} 5 \mathrm{~B}$ & $122.9(5)$ \\
\hline $\mathrm{N} 7 \mathrm{~B}-\mathrm{C} 2 \mathrm{~B}-\mathrm{C} 1 \mathrm{~B}$ & $122.6(6)$ \\
\hline $\mathrm{N} 6 \mathrm{~A}-\mathrm{C} 1 \mathrm{~A}-\mathrm{C} 2 \mathrm{~A}-\mathrm{N} 7 \mathrm{~A}$ & $-3.1(8)$ \\
\hline $\mathrm{C} 5 \mathrm{~A}-\mathrm{C} 1 \mathrm{~A}-\mathrm{C} 2 \mathrm{~A}-\mathrm{N} 7 \mathrm{~A}$ & $174.1(5)$ \\
\hline $\mathrm{N} 7 \mathrm{~A}-\mathrm{C} 3 \mathrm{~A}-\mathrm{C} 4 \mathrm{~A}-\mathrm{N} 6 \mathrm{~A}$ & $-3.0(9)$ \\
\hline $\mathrm{N} 6 \mathrm{~A}-\mathrm{C} 1 \mathrm{~A}-\mathrm{C} 5 \mathrm{~A}-\mathrm{N} 1 \mathrm{~A}$ & $173.9(5)$ \\
\hline $\mathrm{C} 2 \mathrm{~A}-\mathrm{C} 1 \mathrm{~A}-\mathrm{C} 5 \mathrm{~A}-\mathrm{N} 1 \mathrm{~A}$ & $-3.5(7)$ \\
\hline $\mathrm{N} 6 \mathrm{~A}-\mathrm{C} 1 \mathrm{~A}-\mathrm{C} 5 \mathrm{~A}-\mathrm{N} 5 \mathrm{~A}$ & $-1.2(7)$ \\
\hline
\end{tabular}

$\begin{array}{ll}\mathrm{N} 7 \mathrm{~B}-\mathrm{C} 3 \mathrm{~B}-\mathrm{H} 3 \mathrm{~B} & 118.4 \\ \mathrm{C} 4 \mathrm{~B}-\mathrm{C} 3 \mathrm{~B}-\mathrm{H} 3 \mathrm{~B} & 118.4 \\ \mathrm{~N} 6 \mathrm{~B}-\mathrm{C} 4 \mathrm{~B}-\mathrm{C} 3 \mathrm{~B} & 121.1(5) \\ \mathrm{N} 6 \mathrm{~B}-\mathrm{C} 4 \mathrm{~B}-\mathrm{H} 4 \mathrm{~B} & 119.5 \\ \mathrm{C} 3 \mathrm{~B}-\mathrm{C} 4 \mathrm{~B}-\mathrm{H} 4 \mathrm{~B} & 119.5 \\ \mathrm{~N} 1 \mathrm{~B}-\mathrm{C} 5 \mathrm{~B}-\mathrm{N} 5 \mathrm{~B} & 108.6(4) \\ \mathrm{N} 1 \mathrm{~B}-\mathrm{C} 5 \mathrm{~B}-\mathrm{C} 1 \mathrm{~B} & 123.8(5) \\ \mathrm{N} 5 \mathrm{~B}-\mathrm{C} 5 \mathrm{~B}-\mathrm{C} 1 \mathrm{~B} & 127.5(4) \\ \mathrm{N} 2 \mathrm{~B}-\mathrm{C} 6 \mathrm{~B}-\mathrm{N} 5 \mathrm{~B} & 110.8(4) \\ \mathrm{N} 2 \mathrm{~B}-\mathrm{C} 6 \mathrm{~B}-\mathrm{N} 3 \mathrm{~B} & 143.1(5) \\ \mathrm{N} 5 \mathrm{~B}-\mathrm{C} 6 \mathrm{~B}-\mathrm{N} 3 \mathrm{~B} & 106.1(4) \\ \mathrm{N} 4 \mathrm{~B}-\mathrm{C} 7 \mathrm{~B}-\mathrm{N} 3 \mathrm{~B} & 113.8(4) \\ \mathrm{N} 4 \mathrm{~B}-\mathrm{C} 7 \mathrm{~B}-\mathrm{C} 8 \mathrm{~B} & 124.0(5) \\ \mathrm{N} 3 \mathrm{~B}-\mathrm{C} 7 \mathrm{~B}-\mathrm{C} 8 \mathrm{~B} & 122.2(5) \\ \mathrm{C} 7 \mathrm{~B}-\mathrm{C} 8 \mathrm{~B}-\mathrm{H} D & 109.5\end{array}$

$\mathrm{C} 7 \mathrm{~B}-\mathrm{C} 8 \mathrm{~B}-\mathrm{H} 8 \mathrm{D} \quad 109.5$

$\mathrm{C} 7 \mathrm{~B}-\mathrm{C} 8 \mathrm{~B}-\mathrm{H} 8 \mathrm{E} \quad 109.5$

$\mathrm{H} 8 \mathrm{D}-\mathrm{C} 8 \mathrm{~B}-\mathrm{H} 8 \mathrm{E} \quad 109.5$

$\mathrm{C} 7 \mathrm{~B}-\mathrm{C} 8 \mathrm{~B}-\mathrm{H} 8 \mathrm{~F} \quad 109.5$

$\mathrm{H} 8 \mathrm{D}-\mathrm{C} 8 \mathrm{~B}-\mathrm{H} 8 \mathrm{~F} \quad 109.5$

$\mathrm{H} 8 \mathrm{E}-\mathrm{C} 8 \mathrm{~B}-\mathrm{H} 8 \mathrm{~F} \quad 109.5$

$\mathrm{C} 5 \mathrm{~B}-\mathrm{N} 1 \mathrm{~B}-\mathrm{N} 2 \mathrm{~B} \quad 109.4$ (4)

$\mathrm{C} 6 \mathrm{~B}-\mathrm{N} 2 \mathrm{~B}-\mathrm{N} 1 \mathrm{~B} \quad 105.0(4)$

$\mathrm{C} 6 \mathrm{~B}-\mathrm{N} 2 \mathrm{~B}-\mathrm{Zn} \quad 139.0(3)$

$\mathrm{N} 1 \mathrm{~B}-\mathrm{N} 2 \mathrm{~B}-\mathrm{Zn} \quad 115.6(3)$

$\mathrm{C} 6 \mathrm{~B}-\mathrm{N} 3 \mathrm{~B}-\mathrm{C} 7 \mathrm{~B} \quad 105.6$ (4)

$\mathrm{C} 6 \mathrm{~B}-\mathrm{N} 3 \mathrm{~B}-\mathrm{H} 3 \mathrm{NB} \quad 128(4)$

$\mathrm{C} 7 \mathrm{~B}-\mathrm{N} 3 \mathrm{~B}-\mathrm{H} 3 \mathrm{NB} \quad 125$ (4)

C7B-N4B-N5B 101.7 (4)

$\mathrm{C} 6 \mathrm{~B}-\mathrm{N} 5 \mathrm{~B}-\mathrm{C} 5 \mathrm{~B} \quad 106.2(4)$

C6B-N5B-N4B $112.9(4)$

$\mathrm{C} 5 \mathrm{~B}-\mathrm{N} 5 \mathrm{~B}-\mathrm{N} 4 \mathrm{~B} \quad 140.8(4)$

$\mathrm{C} 1 \mathrm{~B}-\mathrm{N} 6 \mathrm{~B}-\mathrm{C} 4 \mathrm{~B} \quad 115.7(5)$

$\mathrm{C} 2 \mathrm{~B}-\mathrm{N} 7 \mathrm{~B}-\mathrm{C} 3 \mathrm{~B} \quad 115.2(6)$

$\mathrm{N} 2 \mathrm{~B}-\mathrm{Zn}-\mathrm{N} 3 \mathrm{~A} \quad 105.76(17)$

$\mathrm{N} 2 \mathrm{~B}-\mathrm{Zn}-\mathrm{Br} 2 \quad 106.45(13)$

$\mathrm{N} 3 \mathrm{~A}-\mathrm{Zn}-\mathrm{Br} 2 \quad 118.70$ (12)

$\mathrm{N} 2 \mathrm{~B}-\mathrm{Zn}-\mathrm{Br} 1 \quad 109.44$ (14)

$\mathrm{N} 3 \mathrm{~A}-\mathrm{Zn}-\mathrm{Br} 1 \quad 102.56(12)$

$\mathrm{Br} 2-\mathrm{Zn}-\mathrm{Br} 1$

H1W-O1W-H2W 107.6

$\begin{array}{ll}\mathrm{N} 6 \mathrm{~B}-\mathrm{C} 1 \mathrm{~B}-\mathrm{C} 2 \mathrm{~B}-\mathrm{N} 7 \mathrm{~B} & 4.8(10) \\ \mathrm{C} 5 \mathrm{~B}-\mathrm{C} 1 \mathrm{~B}-\mathrm{C} 2 \mathrm{~B}-\mathrm{N} 7 \mathrm{~B} & -175.6(6) \\ \mathrm{N} 7 \mathrm{~B}-\mathrm{C} 3 \mathrm{~B}-\mathrm{C} 4 \mathrm{~B}-\mathrm{N} 6 \mathrm{~B} & -0.2(10) \\ \mathrm{N} 6 \mathrm{~B}-\mathrm{C} 1 \mathrm{~B}-\mathrm{C} 5 \mathrm{~B}-\mathrm{N} 1 \mathrm{~B} & -0.6(7) \\ \mathrm{C} 2 \mathrm{~B}-\mathrm{C} 1 \mathrm{~B}-\mathrm{C} 5 \mathrm{~B}-\mathrm{N} 1 \mathrm{~B} & 179.8(5) \\ \mathrm{N} 6 \mathrm{~B}-\mathrm{C} 1 \mathrm{~B}-\mathrm{C} 5 \mathrm{~B}-\mathrm{N} 5 \mathrm{~B} & -178.4(5)\end{array}$




$\begin{array}{ll}\text { C2A-C1A-C5A-N5A } & -178.6(5) \\ \text { N5A-C5A-N1A-N2A } & -0.3(5) \\ \text { C1A-C5A-N1A-N2A } & -176.1(4) \\ \text { N3A-C6A-N2A-N1A } & 178.4(6) \\ \text { N5A-C6A-N2A-N1A } & -0.9(6) \\ \text { C5A-N1A-N2A-C6A } & 0.7(6) \\ \text { N2A-C6A-N3A-C7A } & -178.7(7) \\ \text { N5A-C6A-N3A-C7A } & 0.6(5) \\ \text { N2A-C6A-N3A-Zn } & -12.6(10) \\ \text { N5A-C6A-N3A-Zn } & 166.7(3) \\ \text { N4A-C7A-N3A-C6A } & -0.4(6) \\ \text { C8A-C7A-N3A-C6A } & 178.6(5) \\ \text { N4A-C7A-N3A-Zn } & -168.0(4) \\ \text { C8A-C7A-N3A-Zn } & 11.1(7) \\ \text { N3A-C7A-N4A-N5A } & 0.1(6) \\ \text { C8A-C7A-N4A-N5A } & -178.9(5) \\ \text { N3A-C6A-N5A-C5A } & -178.9(4) \\ \text { N2A-C6A-N5A-C5A } & 0.7(5) \\ \text { N3A-C6A-N5A-N4A } & -0.6(5) \\ \text { N2A-C6A-N5A-N4A } & 179.0(4) \\ \text { N1A-C5A-N5A-C6A } & -0.3(5) \\ \text { C1A-C5A-N5A-C6A } & 175.3(5) \\ \text { N1A-C5A-N5A-N4A } & -177.7(5) \\ \text { C1A-C5A-N5A-N4A } & -2.1(9) \\ \text { C7A-N4A-N5A-C6A } & 0.3(5) \\ \text { C7A-N4A-N5A-C5A } & 177.6(6) \\ \text { C3A-C4A-N6A-C1A } & 2.0(8) \\ \text { C2A-C1A-N6A-C4A } & 1.0(7) \\ \text { C5A-C1A-N6A-C4A } & -176.3(5) \\ \text { C1A-C2A-N7A-C3A } & 2.1(8) \\ \text { C4A-C3A-N7A-C2A } & 0.8(8) \\ & \end{array}$

$\begin{array}{ll}\mathrm{C} 2 \mathrm{~B}-\mathrm{C} 1 \mathrm{~B}-\mathrm{C} 5 \mathrm{~B}-\mathrm{N} 5 \mathrm{~B} & 2.0(8) \\ \mathrm{N} 5 \mathrm{~B}-\mathrm{C} 5 \mathrm{~B}-\mathrm{N} 1 \mathrm{~B}-\mathrm{N} 2 \mathrm{~B} & 1.5(6) \\ \mathrm{C} 1 \mathrm{~B}-\mathrm{C} 5 \mathrm{~B}-\mathrm{N} 1 \mathrm{~B}-\mathrm{N} 2 \mathrm{~B} & -176.7(4) \\ \mathrm{N} 5 \mathrm{~B}-\mathrm{C} 6 \mathrm{~B}-\mathrm{N} 2 \mathrm{~B}-\mathrm{N} 1 \mathrm{~B} & -0.9(6) \\ \mathrm{N} 3 \mathrm{~B}-\mathrm{C} 6 \mathrm{~B}-\mathrm{N} 2 \mathrm{~B}-\mathrm{N} 1 \mathrm{~B} & 176.8(7) \\ \mathrm{N} 5 \mathrm{~B}-\mathrm{C} 6 \mathrm{~B}-\mathrm{N} 2 \mathrm{~B}-\mathrm{Zn} & 172.4(4) \\ \mathrm{N} 3 \mathrm{~B}-\mathrm{C} 6 \mathrm{~B}-\mathrm{N} 2 \mathrm{~B}-\mathrm{Zn} & -9.9(12) \\ \mathrm{C} 5 \mathrm{~B}-\mathrm{N} 1 \mathrm{~B}-\mathrm{N} 2 \mathrm{~B}-\mathrm{C} 6 \mathrm{~B} & -0.4(6) \\ \mathrm{C} 5 \mathrm{~B}-\mathrm{N} 1 \mathrm{~B}-\mathrm{N} 2 \mathrm{~B}-\mathrm{Zn} & -175.5(3) \\ \mathrm{N} 2 \mathrm{~B}-\mathrm{C} 6 \mathrm{~B}-\mathrm{N} 3 \mathrm{~B}-\mathrm{C} 7 \mathrm{~B} & -177.2(7) \\ \mathrm{N} 5 \mathrm{~B}-\mathrm{C} 6 \mathrm{~B}-\mathrm{N} 3 \mathrm{~B}-\mathrm{C} 7 \mathrm{~B} & 0.5(5) \\ \mathrm{N} 4 \mathrm{~B}-\mathrm{C} 7 \mathrm{~B}-\mathrm{N} 3 \mathrm{~B}-\mathrm{C} 6 \mathrm{~B} & -0.9(6) \\ \mathrm{C} 8 \mathrm{~B}-\mathrm{C} 7 \mathrm{~B}-\mathrm{N} 3 \mathrm{~B}-\mathrm{C} 6 \mathrm{~B} & 178.7(5) \\ \mathrm{N} 3 \mathrm{~B}-\mathrm{C} 7 \mathrm{~B}-\mathrm{N} 4 \mathrm{~B}-\mathrm{N} 5 \mathrm{~B} & 0.8(5) \\ \mathrm{C} 8 \mathrm{~B}-\mathrm{C} 7 \mathrm{~B}-\mathrm{N} 4 \mathrm{~B}-\mathrm{N} 5 \mathrm{~B} & -178.7(5) \\ \mathrm{N} 2 \mathrm{~B}-\mathrm{C} 6 \mathrm{~B}-\mathrm{N} 5 \mathrm{~B}-\mathrm{C} 5 \mathrm{~B} & 1.8(6) \\ \mathrm{N} 3 \mathrm{~B}-\mathrm{C} 6 \mathrm{~B}-\mathrm{N} 5 \mathrm{~B}-\mathrm{C} 5 \mathrm{~B} & -176.8(4) \\ \mathrm{N} 2 \mathrm{~B}-\mathrm{C} 6 \mathrm{~B}-\mathrm{N} 5 \mathrm{~B}-\mathrm{N} 4 \mathrm{~B} & 178.5(4) \\ \mathrm{N} 3 \mathrm{~B}-\mathrm{C} 6 \mathrm{~B}-\mathrm{N} 5 \mathrm{~B}-\mathrm{N} 4 \mathrm{~B} & -0.1(6) \\ \mathrm{N} 1 \mathrm{~B}-\mathrm{C} 5 \mathrm{~B}-\mathrm{N} 5 \mathrm{~B}-\mathrm{C} 6 \mathrm{~B} & -2.0(5) \\ \mathrm{C} 1 \mathrm{~B}-\mathrm{C} 5 \mathrm{~B}-\mathrm{N} 5 \mathrm{~B}-\mathrm{C} 6 \mathrm{~B} & 176.1(5) \\ \mathrm{N} 1 \mathrm{~B}-\mathrm{C} 5 \mathrm{~B}-\mathrm{N} 5 \mathrm{~B}-\mathrm{N} 4 \mathrm{~B} & -177.2(5) \\ \mathrm{C} 1 \mathrm{~B}-\mathrm{C} 5 \mathrm{~B}-\mathrm{N} 5 \mathrm{~B}-\mathrm{N} 4 \mathrm{~B} & 0.9(10) \\ \mathrm{C} 7 \mathrm{~B}-\mathrm{N} 4 \mathrm{~B}-\mathrm{N} 5 \mathrm{~B}-\mathrm{C} 6 \mathrm{~B} & -0.4(5) \\ \mathrm{C} 7 \mathrm{~B}-\mathrm{N} 4 \mathrm{~B}-\mathrm{N} 5 \mathrm{~B}-\mathrm{C} 5 \mathrm{~B} & 174.6(6) \\ \mathrm{C} 2 \mathrm{~B}-\mathrm{C} 1 \mathrm{~B}-\mathrm{N} 6 \mathrm{~B}-\mathrm{C} 4 \mathrm{~B} & -2.5(8) \\ \mathrm{C} 5 \mathrm{~B}-\mathrm{C} 1 \mathrm{~B}-\mathrm{N} 6 \mathrm{~B}-\mathrm{C} 4 \mathrm{~B} & 177.8(5) \\ \mathrm{C} 3 \mathrm{~B}-\mathrm{C} 4 \mathrm{~B}-\mathrm{N} 6 \mathrm{~B}-\mathrm{C} 1 \mathrm{~B} & 0.3(8) \\ \mathrm{C} 1 \mathrm{~B}-\mathrm{C} 2 \mathrm{~B}-\mathrm{N} 7 \mathrm{~B}-\mathrm{C} 3 \mathrm{~B} & -4.3(10) \\ \mathrm{C} 4 \mathrm{~B}-\mathrm{C} 3 \mathrm{~B}-\mathrm{N} 7 \mathrm{~B}-\mathrm{C} 2 \mathrm{~B} & 2.1(10) \\ & \end{array}$

Hydrogen-bond geometry $\left(A,{ }^{\circ}\right)$

\begin{tabular}{lllll}
\hline$D-\mathrm{H} \cdots A$ & $D-\mathrm{H}$ & $\mathrm{H} \cdots A$ & $D \cdots A$ & $D-\mathrm{H} \cdots A$ \\
\hline $\mathrm{C} 2 A-\mathrm{H} 2 A \cdots \mathrm{Br} 1^{\mathrm{i}}$ & 0.93 & 3.13 & $3.995(6)$ & 155 \\
$\mathrm{~N} 2 A-\mathrm{H} 2 N A \cdots \mathrm{N} 1 B$ & $0.84(2)$ & $2.09(4)$ & $2.786(6)$ & $139(5)$ \\
$\mathrm{N} 2 A-\mathrm{H} 2 N A \cdots \mathrm{N} 6 B$ & $0.84(2)$ & $2.39(4)$ & $3.126(6)$ & $145(5)$ \\
$\mathrm{C} 2 B-\mathrm{H} 2 B \cdots \mathrm{N} 4 B$ & 0.93 & 2.62 & $3.276(7)$ & 129 \\
$\mathrm{C} 4 B-\mathrm{H} 4 B \cdots \mathrm{Br} 1^{\mathrm{i}}$ & 0.93 & 2.86 & $3.791(6)$ & 176 \\
$\mathrm{~N} 3 B-\mathrm{H} 3 N B \cdots \mathrm{O} 1 W$ & $0.85(2)$ & $1.85(3)$ & $2.669(6)$ & $163(6)$ \\
$\mathrm{O} 1 W-\mathrm{H} 1 W \cdots \mathrm{Br} 1^{\mathrm{ii}}$ & 1.03 & 2.39 & $3.375(6)$ & 162 \\
$\mathrm{O} 1 W-\mathrm{H} 2 W \cdots \mathrm{N} 7 A^{\mathrm{ii}}$ & 0.98 & 2.03 & $2.901(7)$ & 148
\end{tabular}

Symmetry codes: (i) $-x+1,-y,-z+1$; (ii) $-x,-y+1,-z+1$; (iii) $x-1, y+1, z$. 